\title{
NGC 362: another globular cluster with a split red giant branch $\star, \star \star, \star \star \star$
}

\author{
E. Carretta ${ }^{1}$, A. Bragaglia ${ }^{1}$, R. G. Gratton ${ }^{2}$, S. Lucatello ${ }^{2}$, V. D’Orazi ${ }^{3,4}$, M. Bellazzini ${ }^{1}$, G. Catanzaro ${ }^{5}$, F. Leone ${ }^{6}$, \\ Y. Momany ${ }^{2,7}$, and A. Sollima ${ }^{1}$
}

\author{
1 INAF - Osservatorio Astronomico di Bologna, via Ranzani 1, 40127 Bologna, Italy \\ e-mail: eugenio.carretta@oabo.inaf.it \\ 2 INAF - Osservatorio Astronomico di Padova, Vicolo dell'Osservatorio 5, 35122 Padova, Italy \\ 3 Dept. of Physics and Astronomy, Macquarie University, Sydney, NSW, 2109 Australia \\ ${ }^{4}$ Monash Centre for Astrophysics, Monash University, School of Mathematical Sciences, Building 28, Clayton VIC 3800 , \\ Melbourne, Australia \\ 5 INAF - Osservatorio Astrofisico di Catania, via S. Sofia 78, 95123 Catania, Italy \\ ${ }_{7}^{6}$ Dipartimento di Fisica e Astronomia, Università di Catania, via S. Sofia 78, 95123 Catania, Italy \\ 7 European Southern Observatory, Alonso de Cordova 3107, Vitacura, Santiago, Chile
}

Received 16 May 2013 / Accepted 11 July 2013

\section{ABSTRACT}

\begin{abstract}
We obtained FLAMES GIRAFFE+UVES spectra for both first- and second-generation red giant branch (RGB) stars in the globular cluster (GC) NGC 362 and used them to derive abundances of 21 atomic species for a sample of 92 stars. The surveyed elements include proton-capture $(\mathrm{O}, \mathrm{Na}, \mathrm{Mg}, \mathrm{Al}, \mathrm{Si}), \alpha$-capture $(\mathrm{Ca}, \mathrm{Ti}), \mathrm{Fe}-$ peak $(\mathrm{Sc}, \mathrm{V}, \mathrm{Mn}, \mathrm{Co}, \mathrm{Ni}, \mathrm{Cu})$, and neutron-capture elements (Y, Zr, $\mathrm{Ba}, \mathrm{La}, \mathrm{Ce}, \mathrm{Nd}, \mathrm{Eu}, \mathrm{Dy})$. The analysis is fully consistent with that presented for twenty GCs in previous papers of this series. Stars in NGC 362 seem to be clustered into two discrete groups along the $\mathrm{Na}-\mathrm{O}$ anti-correlation with a gap at $[\mathrm{O} / \mathrm{Na}] \sim 0$ dex. Na-rich, second generation stars show a trend to be more centrally concentrated, although the level of confidence is not very high. When compared to the classical second-parameter twin NGC 288 with similar metallicity but different horizontal branch type and a much lower total mass, the proton-capture processing in stars of NGC 362 seems to be more extreme, confirming previous analysis. We discovered the presence of a secondary RGB sequence, which is redder than the bulk of the RGB. A preliminary estimate shows that this sequence comprises about $6 \%$ of RGB stars. Our spectroscopic data and literature photometry indicate that this sequence is populated almost exclusively by giants rich in $\mathrm{Ba}$ and is probably rich in all s-process elements, as found in other clusters. In this regard, NGC 362 joins previously studied GCs like NGC 1851, NGC 6656 (M 22), and NGC 7089 (M 2).
\end{abstract}

Key words. stars: Population II - globular clusters: general - stars: abundances - stars: atmospheres - globular clusters: individual: NGC 362

\section{Introduction}

In the past few years, the paradigm of multiple stellar populations as a basic ingredient of Galactic globular clusters (GCs) has become well assessed (see the review by Gratton et al. 2004 and the recent updates by Martell 2011 and Gratton et al. 2012, which also include references to the photometric evidence). At least two bursts of star formation must have occurred in GCs with the second generation being formed from a mix of pristine gas and matter enriched by nuclear processing in the massive stars of the first generation (see Gratton et al. 2001). The impact of this chain of events is not limited to the history of GCs, but it probably had a key role in the building of a considerable fraction of the Galactic halo (Carretta et al. 2010a; Vesperini et al. 2010; Martell et al. 2011). Some important links to global cluster parameters (e.g., total mass, age, and location in the

\footnotetext{
* Based on observations collected at ESO telescopes under programme 083.D-0208.

$\star \star$ Appendix A is available in electronic form at

http://www . aanda.org

$\star \star \star$ Full Tables 2-8 are only available at the CDS via anonymous ftp to cdsarc.u-strasbg.fr (130.79.128.5) or via

http://cdsarc.u-strasbg.fr/viz-bin/qcat?]/A+A/557/A138
}

Galaxy, Carretta et al. 2010a) were discovered, and the ouput of extensive surveys performed with multi-object facilities, like FLAMES, (see Carretta et al. 2006, 2009a,b,c) pointed out that the characteristics of the nuclear processing occurring in early phases differ from cluster to cluster. This evidence calls for an in-depth investigation of a number of GCs with different parameters, like metallicity, concentration, horizontal branch $(\mathrm{HB})$ morphology. This was the original motivation of our ongoing FLAMES survey (see Carretta et al. 2006).

In this paper, we present an extensive analysis of chemical abundances of multiple stellar populations in the GC NGC 362. This is a moderately metal-rich object $\left([\mathrm{Fe} / \mathrm{H}]=-1.26 \mathrm{dex}^{1}\right.$ in the 2011 update of the Harris 1996 catalogue, H96 hereinafter, which is based on the metallicity scale of Carretta et al. 2009c) seen projected toward the Small Magellanic Cloud.

The globular cluster NGC 362 is variously classified as a red HB (RHB), a young halo (Mackey and van den Bergh 2005), or an inner halo (Carretta et al. 2010a) cluster. It belongs to a group of GCs with low total orbital energy (small orbit sizes) and

1 We adopt the usual spectroscopic notation, i.e. $[\mathrm{X}]=\log (\mathrm{X})_{\mathrm{star}}-$ $\log (\mathrm{X})_{\odot}$ for any abundance quantity $\mathrm{X}$, and $\log \epsilon(\mathrm{X})=\log \left(N_{\mathrm{X}} / N_{\mathrm{H}}\right)+$ 12.0 for absolute number density abundances. 
unusual orbital parameters. The orbit has a chaotic behaviour and is confined close to the Galactic plane $\left(z_{\max }=2.1 \mathrm{kpc}\right)$ with high eccentricity and a small inclination off the orbital plane (Dinescu et al. 1999). An extensive variability survey in NGC 362 was made by Székely et al. (2007), who found more than $45 \mathrm{RR}$ Lyrae variables, a metallicity $[\mathrm{Fe} / \mathrm{H}]=-1.16 \mathrm{dex}$, and a mean period of RRab stars of $0.585 \pm 0.081$ days, which places this cluster in the Oosterhoff type I group.

The pair NGC 362-NGC 288 is considered a classical example of second parameter clusters since the colour of the $\mathrm{HB}$ of these two GCs is very different (NGC 362: red; NGC 288: blue) despite their similar metal-abundance. Catelan et al. (2001) compared the relative age provided by the HB morphology with that obtained from the main sequence for the pair NGC 362-NGC 288. They used the bimodal HB of NGC 1851 as a "bridge" (see also Bellazzini et al. 2001). They found that NGC 362 is about 2 Gyr younger than NGC 288, supporting the concept that age is a main second parameter (beside metallicity) in shaping the distribution of stars along the HB. The same result was obtained by Dotter et al. (2010) and Gratton et al. (2010) from the analysis of homogeneous photometric and spectroscopic databases. While Catelan et al. claimed that the mass dispersion on the HB of NGC 362 is substantially larger than for NGC 288, the opposite however was found by Gratton et al. According to their analysis, the mass spread required to account for the HB morphology in NGC 362 is only $0.004 M_{\odot}$ against $0.024 M_{\odot}$ for NGC 288. This is due to the adoption of an universal mass loss law with a linear dependence on metallicity that well reproduces the median colours of HB stars.

Piotto et al. (2012) found that a few (actually, less then 3\%) subgiant branch stars of NGC 362 are fainter than the other subgiants of a similar colour. This faint sequence might be interpreted as a small group of objects having either older ages or a larger total CNO content than the majority of the stars.

Abundance analyses of stars in NGC 362 are scanty. Several studies based on low dispersion spectroscopy determined the pattern of the $\mathrm{CN}$ and $\mathrm{CH}$ distribution in NGC 362, typically found to be bimodal (Smith 1983, 1984; Kayser et al. 2008; Smith \& Langland-Shula 2009, and reference therein). Shetrone \& Keane (2000) derived abundances for several elements in a dozen of giants from high dispersion spectra and compared their chemical pattern to that derived from 13 giants in NGC 288. At the epoch (before the analysis of $\mathrm{O}, \mathrm{Na}, \mathrm{Mg}$, and $\mathrm{Al}$ in unevolved cluster stars by Gratton et al. 2001), it was still debated how much evolutionary (mixing) effects were superimposed to ab initio abundance variations. Even from their moderate-size samples, Shetrone \& Keane (2000) were able to point out differences in the zero-point and slope existing among the observed $\mathrm{Na}-\mathrm{O}$ anti-correlation in this pair of GCs. Recently, Worley \& Cottrell (2010) used high dispersion spectroscopy to study the pattern of light and heavy neutron-capture process elements in NGC 362. In our study, we enlarge the sample of stars analysed with moderate-high resolution spectroscopy to more than 90 red giant branch (RGB) stars in NGC 362, obtaining homogeneous abundances of proton-capture, $\alpha$-capture, Fe-peak, and neutroncapture elements.

The paper is organized as follows. Observations are presented in Sect. 2; radial velocities and a brief discussion of the kinematics of the cluster are in Sect. 3. Section 4 presents the derivation of the atmospheric parameters and the abundance analysis; results of this analysis are given in Sect. 5. Finally, discussion and conclusions are in Sect. 6. The appendix presents the derivation of errors in the abundance analysis.

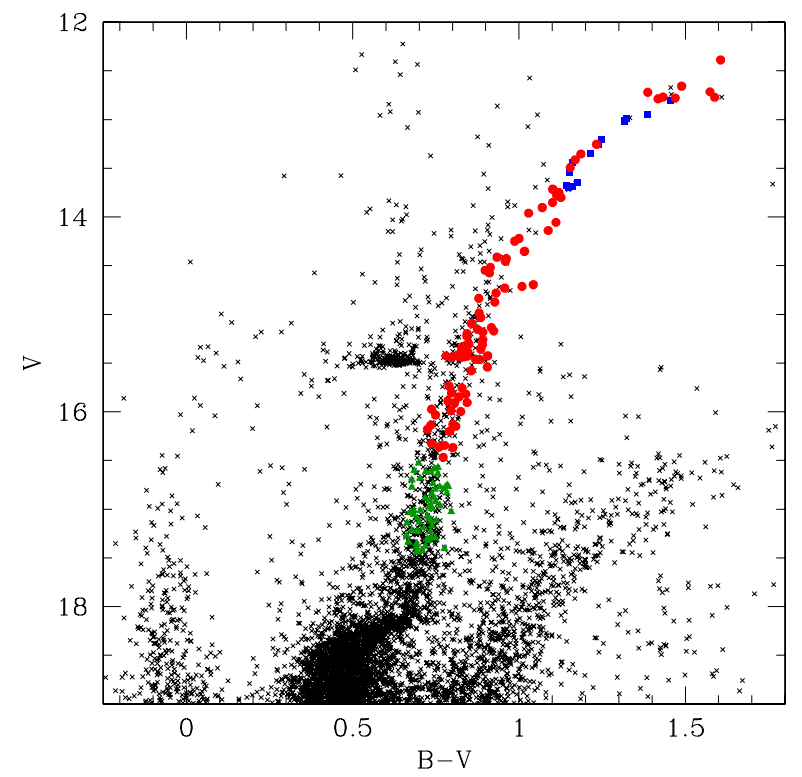

Fig. 1. Colour-magnitude diagram (CMD) $V, B-V$ of NGC 362 (light grey crosses). Stars selected for the present study are plotted as filled, larger symbols: blue squares are the stars observed with UVES, red circles are stars with GIRAFFE spectra, and green triangles are stars observed with GIRAFFE but not analyzed (see text). The faint blue and red sequences are due to the young main sequence stars and old red giants of the Small Magellanic Cloud.

Table 1. Log of FLAMES observations for NGC 362.

\begin{tabular}{lccccc}
\hline \hline Date & UT & $\begin{array}{c}\text { Exp. } \\
(\mathrm{s})\end{array}$ & Grating & $\begin{array}{c}\text { Seeing } \\
\left({ }^{\prime \prime}\right)\end{array}$ & Airmass \\
\hline Aug. 04, 2009 & $08: 07: 18.230$ & 2600 & HR11 & 1.02 & 1.454 \\
Aug. 04, 2009 & $08: 57: 24.456$ & 2600 & HR11 & 0.88 & 1.442 \\
Aug. 05, 2009 & $07: 27: 25.601$ & 2600 & HR13 & 0.65 & 1.482 \\
Aug. 05, 2009 & $08: 12: 33.313$ & 2600 & HR13 & 0.86 & 1.449 \\
\hline
\end{tabular}

\section{Observations}

Our targets were selected from unpublished Johnson $B, V$ Wide Field Imager (WFI) photometry, which was obtained at the $2.2 \mathrm{~m}$ ESO-Max Planck Telescope (La Silla, Chile), and these objects positions were derived by us (Y. Momany). We then integrated the photometry of the targets with $K$ band magnitudes from the Point Source Catalogue of 2MASS (Skrutskie et al. 2006). The $V, B-V$ colour magnitude diagram (CMD) of NGC 362 is shown in Fig. 1 with superimposed stars of our spectroscopic sample, which are chosen to be near the RGB ridge line and with no close companion. NGC 362 is seen projected against the Small Magellanic Cloud, which is responsible for the faint blue and red sequences visible in that diagram; they are young main sequence stars and old red giants, respectively.

The log of the observations is given in Table 1. We obtained two exposures with the HR11 high-resolution grating that covers the Na I 5682-88 $⿱$ doublet and two exposures with the HR13 grating that includes the [O I] forbidden lines at 6300-63 $\AA$. We observed a total of 14 (bright) giants with the fibres that feed the UVES spectrograph (Red Arm, with spectral range from 4800 to $6800 \AA$ and $R=45000$; blue squares in Fig. 1) and 136 RGB stars with GIRAFFE (filled circles and triangles). All stars turned out (likely) to be members of the cluster based on radial velocities (RV). However, we retained only stars with effective temperature below $5200 \mathrm{~K}$ for the present analysis, 
Table 2. List and relevant information for target stars in NGC 362.

\begin{tabular}{lccccccc}
\hline \hline ID & RA & Dec & $B$ & $V$ & $K$ & $R V($ Hel $)$ & Notes \\
\hline 986 & 10320.037 & -704955.53 & 15.227 & 14.139 & 11.443 & 228.83 & HR11, HR13 \\
995 & 10323.159 & -704954.98 & 15.446 & 14.548 & 12.013 & 222.77 & HR13 \\
\hline
\end{tabular}

Notes. The complete table is available electronically at the CDS.

because the low $\mathrm{S} / \mathrm{N}$ and noisy spectra prevented us from measuring accurate enough equivalent widths (EWs) for warmer stars (green triangles) ${ }^{2}$. This limit corresponds to a magnitude level $V=16.47$.

We used the 1D, wavelength-calibrated spectra that were reduced by the ESO personnel with the dedicated FLAMES pipeline. Radial velocities (RV) for stars observed with the GIRAFFE spectrograph were obtained using the IRAF $^{3}$ task FXCORR with appropriate templates, while RVs for the stars observed with UVES were derived with the IRAF task RVIDLINES.

The star 13875 observed with GIRAFFE shows the TiO band head at $6158 \AA$ in the HR13 spectrum (see Valenti et al. 1998). This star has many peculiarities, beside being of spectral type M. It is a variable (V2: Clement 1997) with a period of 90 days, was found to be very Li-rich by Smith et al. (1999), and presents carbon dust, according to Boyer et al. (2009). It was discarded from the following analysis.

We have 12 stars in common between the UVES and GIRAFFE datasets; disregarding star V2, our final sample in NGC 362 consists of 92 RGB stars. Coordinates, magnitudes, and heliocentric RVs are shown in Table 2 (The full table is only available in electronic form at CDS).

\section{Kinematics}

As discussed in Bellazzini et al. (2012; B12 hereinafter), the samples of RV estimates that are obtained as a natural by product of this project are not ideal for kinematical analyses. Because of the fibre allocation constraints described in B12, they are, however, nicely complementary to existing datasets in many cases, since they preferentially probe the outskirts of the clusters. This allowed us to reveal rotation signals in the outer regions of several clusters that went unnoticed in previous studies. As for the chemical analysis, we use only stars cooler than $5200 \mathrm{~K}$ (red circles in Fig. 1 and below) in the following since the membership only for these stars is fully ascertained based on both RV and chemical composition. Stars warmer than this value are plotted as green circles (as in Fig. 1) in the figures presenting kinematic results for completeness. We perform the same kind of analysis of B12. We refer to that paper for details and discussion.

The detailed analysis by Fischer et al. (1993: F93) is based on 210 RV members of this cluster: all of them lie within $R=4.0^{\prime}$. On the other hand, our sample covers the range of $1.0^{\prime} \leq R \leq 11.0^{\prime}$, reaching the tidal radius of the clusters $\left(r_{\mathrm{t}}=10.3^{\prime}, \mathrm{H} 96\right)$. We merged our catalogue with the one by F93, keeping our RV when estimates from both sources are available. From a subset of 38 common stars (excluding two stars, our stars 11413 and 18947 , classified as binary from

\footnotetext{
2 The hottest star left in our final sample is $\# 5447$ with an effective temperature of $5203 \mathrm{~K}$.

3 IRAF is distributed by the National Optical Astronomical Observatory, which is operated by the Association of Universities for Research in Astronomy, under contract with the National Science Foundation.
}

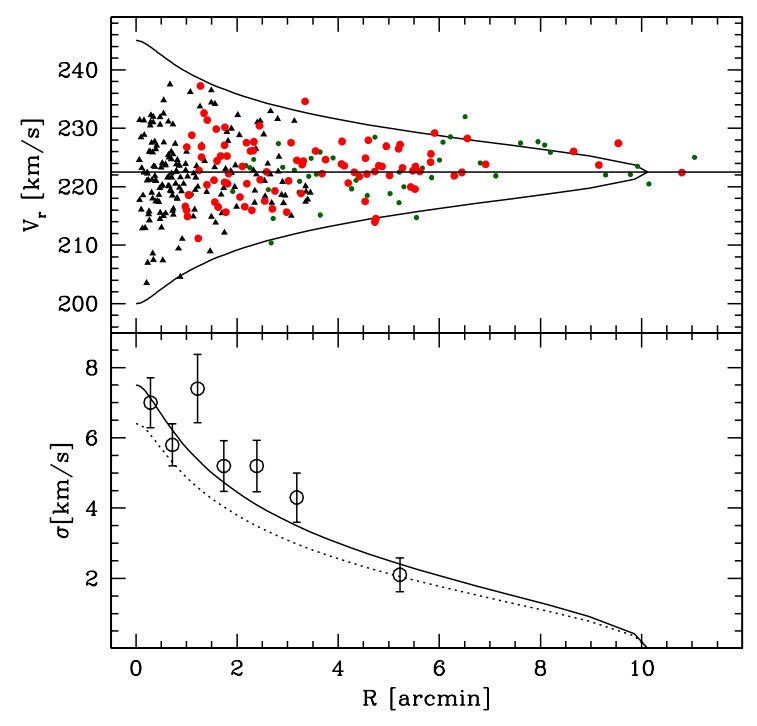

Fig. 2. Radial velocity as a function of the distance from the cluster centre (upper panel). Coloured symbols are the same as in Fig. 1; filled triangles are stars from F93. The solid lines represents the $\pm 3 \sigma$ envelope of the King (1966) model that best fits the surface brightness profile, according to H96. The profile is normalized to the central velocity dispersion $\sigma_{0}=7.5 \mathrm{~km} \mathrm{~s}^{-1}$. Velocity dispersion profile (lower panel) as derived from the global sample (this work + F93, excluding stars lacking abundance estimates). The solid line is the profile of the same King (1966) model as above; the dotted line is the same model normalized at the central velocity dispersion reported in $\mathrm{H} 96, \sigma_{0}=6.4 \mathrm{~km} \mathrm{~s}^{-1}$.

RVs at two epochs by F93 $)^{4}$ ), we find a mean difference of $\Delta R V=-1.15 \mathrm{~km} \mathrm{~s}^{-1}$ with $\sigma=1.54$. This small zero point offset was corrected before merging the two samples. The errors on individual RV estimates from the two datasets are very similar $\left(\simeq 1.0 \mathrm{~km} \mathrm{~s}^{-1}\right)$.

In the lower panel of Fig. 2, we show that the velocity dispersion profile obtained from our global sample is hardly compatible with a King (1966) model that has the best-fit parameters reported in $\mathrm{H} 96$ and that is scaled to the central velocity dispersion reported there for this cluster; i.e., $\sigma_{0}=6.4 \mathrm{~km} \mathrm{~s}^{-1}$. Even adopting a higher value that provides an acceptable fit to the observed profile $\left(\sigma_{0}=7.5 \mathrm{~km} \mathrm{~s}^{-1}\right)$, it can be appreciated that there are obvious members lying outside the $\pm 3-\sigma$ envelope of the model, as seen in the upper panel. This suggests that some unbound or partially-bound extra-tidal stars are present, or that King models are not fully adequate to describe the structure and dynamics of the system (see McLaughlin \& van der Marel 2005; Correnti et al. 2011, for discussion and references).

Looking for rotation in the global sample we find no significant signal, in agreement with F93. If we limit to our sample, we find the $A_{\text {rot }}=2.1 \mathrm{~km} \mathrm{~s}^{-1}$ and PA $=147 \mathrm{deg}$ signal shown

\footnotetext{
4 All the stars classified as binaries by F93 have been excluded from the analysis of the cluster kinematics. The four excluded stars are H1348, H1419, H2205, and H2222 in the nomenclature of F93. H1419 = 18947 and H2205 = 11413 are also included in our own sample and in the following chemical analysis.
} 

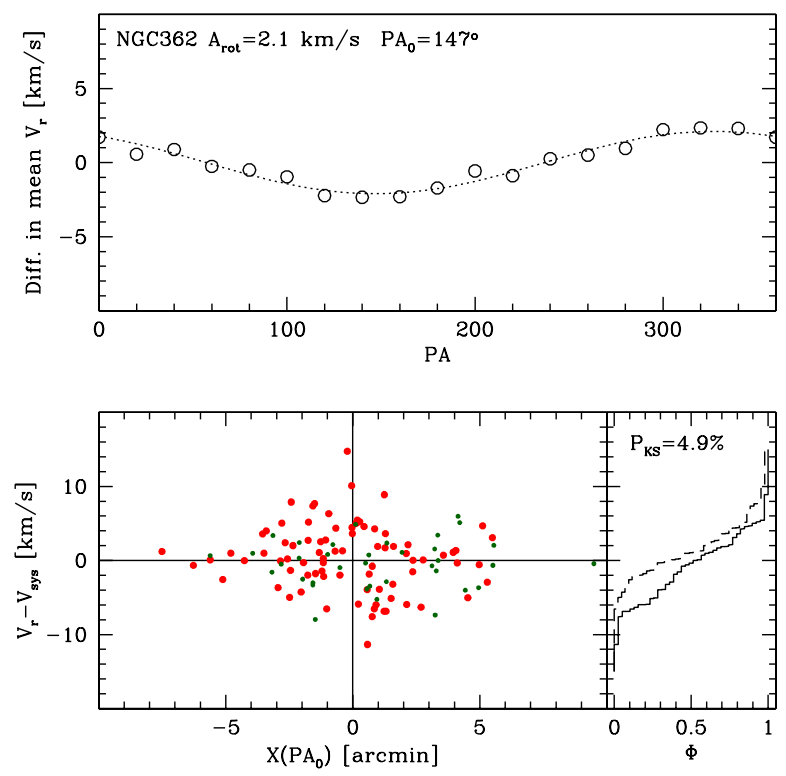

Fig. 3. Rotation in NGC 362. Upper panel: difference between the mean velocities on each side of the cluster with respect to a line passing through the cluster centre with a position angle (PA, measured from north to east, north $=0^{\circ}$, east $=90^{\circ}$ ) as a function of the adopted PA. Our preferred solution is represented by the dotted line and is obtained from bona-fide members from our sample (i.e. stars with abundance estimates). Lower panels: the rotation curve of NGC 362. In the left panel the RV in the system of the cluster is plotted as a function of distance from the centre projected onto the axis perpendicular to the best fit rotation axis found in the upper panel. the meaning of the symbols are the same as in Figs. 1 and 2, above. The right panel shows the comparison of the cumulative $\mathrm{RV}$ distributions of stars having $X\left(\mathrm{PA}_{0}\right)>0.0$ (continuous line) and $X\left(\mathrm{PA}_{0}\right)<0.0$ (dotted line).

in Fig. 3, suggesting that some rotation may be indeed present in the outer regions. Note that the warmer stars excluded from the analysis (green points) appear to share the same pattern. The trend toward $V_{\mathrm{r}}-V_{\text {sys }} \sim 0.0$ at large absolute values of $X\left(\mathrm{PA}_{0}\right)$ is more consistent with an intrinsic nature of the kinematic pattern, as a velocity gradient induced by Galactic tides would show larger amplitudes at longer distances. NGC 362 nicely fits into the correlations recently found by B12.

Finally, we note that the velocity dispersion changes as a function of the $\mathrm{Na}$ abundance: Na-poor stars have lower dispersion than Na-rich ones. This is due to the radial trend shown by the different concentration of Na-poor and $\mathrm{Na}$-rich stars with the first preferentially populating more external regions (see below). There is no significant trend of the rotation pattern with $\mathrm{Na}$ abundance, but it is very likely that our sample is too sparse to investigate these subtle effects.

\section{Atmospheric parameters, abundance analysis, and metallicity}

Only a brief summary of the analysis methods are reported here, since the atmospheric parameters were derived following the same procedure adopted for the other GCs targeted by our FLAMES survey (see e.g. Carretta et al. 2009a,b).

First-pass input effective temperature $T_{\text {eff }}$ (from $V-$ $K$ colours) and bolometric corrections were derived from the calibrations of Alonso et al. (1999, 2001). These values were re- fined using a relation between $T_{\text {eff }}$ and $K$ magnitudes ${ }^{5}$. Gravities were obtained from stellar masses and radii with the latter derived from luminosities and temperatures. The reddening and the distance modulus for NGC 362 were taken from the Harris (1996) catalogue (2011 update). We adopted a mass of $0.85 M_{\odot}$ for all stars, and $M_{\mathrm{bol}, \odot}=4.75$ for the bolometric magnitude for the Sun, as in our previous studies.

The abundance analysis mainly rests on equivalent widths (EWs). We checked that EWs measured on the GIRAFFE spectra are on the same system defined by high-resolution UVES spectra. The values of the microturbulent velocities $v_{\mathrm{t}}$ were obtained by eliminating trends between Fe I abundances and the expected line strength (Magain 1984). Finally, models with the appropriate atmospheric parameters and whose abundances matched those derived from $\mathrm{Fe}$ I lines were interpolated within the Kurucz (1993) grid of model atmospheres (with the option for overshooting on) to derive the final abundances. The adopted atmospheric parameters and iron abundances are listed in Table 3.

Our procedure for error estimates is detailed in Carretta et al. (2009a,b); results with a brief description are given in the appendix for UVES and GIRAFFE observations (see Tables A.1 and A.2, respectively). Oxygen abundances were obtained from the [O I] line at $6300.3 \AA$ (after cleaning this spectral region from telluric lines as described in Carretta et al. 2007a) and from the [O I] line at $6363.8 \AA$, whenever possible. Sodium abundances, derived from the EWs of the 5682-88 and 6154-60 A doublets, were corrected for departures from the LTE assumption following Gratton et al. (1999). Abundances of O, Na, Al (from the 6696-99 A doublet), and Mg (derived as in Carretta et al. 2009b) for individual stars are listed in Table 4.

Beside $\mathrm{Mg}$ (reported among elements involved in protoncapture processes), we measured the abundances of the $\alpha$-elements $\mathrm{Si}, \mathrm{Ca}$, and Ti from either UVES or GIRAFFE spectra (see Table 5) ${ }^{6}$. We measured Ti abundances from lines of both neutral and singly ionised species on UVES spectra (with larger spectral coverage). On average, the abundances obtained from these two ionisation states are in excellent agreement with each other (see below), supporting our adopted scale of atmospheric parameters.

We obtained abundances for the Fe-peak elements Sc II, V I, Cr I, Co I, and Ni I (stars with GIRAFFE and UVES spectra), and additionally $\mathrm{Mn} \mathrm{I}$ and $\mathrm{Cu}$ I for stars with UVES spectra only. The corrections due to the hyperfine structure (see Gratton et al. 2003 for references) were applied whenever relevant (e.g. Sc, V, $\mathrm{Mn}, \mathrm{Co}$ ). We obtained the abundances of several neutron capture elements (Y II, Zr II, Ba II, La II, Ce II, Nd II, Eu II, and Dy II, mostly from UVES spectra), using a combination of spectral synthesis and $E W$ measurements. Details on transitions can be found in Carretta et al. (2011).

Abundances of Fe-peak elements for individual stars are reported in Table 6. Results for neutron capture elements are given in Tables 7 and 8 for stars with UVES and GIRAFFE spectra, respectively. The averages of all measured elements with their rms scatter are listed in Table 9.

The mean metallicity found for NGC 362 from stars with UVES spectra is $[\mathrm{Fe} / \mathrm{H}]=-1.168 \pm 0.014 \pm 0.051 \mathrm{dex}(\mathrm{rms}=$ 0.052 dex, 14 stars), where the first error bar is from statistics and

5 For a few stars with no $K$ in 2MASS, we obtained an estimate of the $K$ values by interpolating a quadratic relation as a function of $V$ magnitudes.

6 The case of $\mathrm{Si}$ as an element involved in the $p$-capture reactions is discussed in Sect. 5.1. 
Table 3. Adopted atmospheric parameters and derived iron abundances.

\begin{tabular}{ccccccccccc}
\hline \hline Star & $\begin{array}{c}T_{\text {eff }} \\
(\mathrm{K})\end{array}$ & $\begin{array}{c}\log g \\
(\mathrm{dex})\end{array}$ & $\begin{array}{c}{[\mathrm{A} / \mathrm{H}]} \\
(\mathrm{dex})\end{array}$ & $\begin{array}{c}v_{\mathrm{t}} \\
\left(\mathrm{km} \mathrm{s}^{-1}\right)\end{array}$ & $\begin{array}{c}{[\mathrm{Fe} / \mathrm{H}] \mathrm{I}} \\
(\mathrm{dex})\end{array}$ & $\mathrm{rms}$ & $n r$ & $\begin{array}{c}{[\mathrm{Fe} / \mathrm{H}] \mathrm{II}} \\
(\mathrm{dex})\end{array}$ & $\mathrm{rms}$ \\
\hline 986 & 4533 & 1.59 & -1.18 & 2.00 & 52 & -1.176 & 0.161 & 3 & -1.141 & 0.164 \\
995 & 4661 & 1.92 & -1.26 & 1.09 & 28 & -1.259 & 0.111 & 3 & -1.154 & 0.028 \\
\hline
\end{tabular}

Notes. The complete table is available electronically at the CDS.

Table 4. Abundances of proton-capture elements in stars of NGC 362. $n$ is the number of lines used in the analysis. Upper limits (limO, Al=0) and detections $(=1)$ for $\mathrm{O}$ and $\mathrm{Al}$ are flagged.

\begin{tabular}{|c|c|c|c|c|c|c|c|c|c|c|c|c|c|c|}
\hline Star & $n$ & {$[\mathrm{O} / \mathrm{Fe}]$} & rms & $n$ & {$[\mathrm{Na} / \mathrm{Fe}]$} & $\mathrm{rms}$ & $n$ & {$[\mathrm{Mg} / \mathrm{Fe}]$} & $\mathrm{rms}$ & $n$ & {$[\mathrm{Al} / \mathrm{Fe}]$} & $\mathrm{rms}$ & LimO & LimAl \\
\hline 986 & 1 & +0.16 & & 4 & +0.21 & 0.12 & 3 & +0.30 & 0.16 & & & & 1 & \\
\hline 995 & 1 & +0.17 & & 2 & -0.14 & 0.04 & & & & & & & 1 & \\
\hline
\end{tabular}

Table 5. Abundances of $\alpha$-elements in stars of NGC 362. $n$ is the number of lines used in the analysis.

\begin{tabular}{ccccccccccccc}
\hline \hline Star & $n$ & {$[\mathrm{Si} / \mathrm{Fe}]$} & $\mathrm{rms}$ & $n$ & {$[\mathrm{Ca} / \mathrm{Fe}]$} & $\mathrm{rms}$ & $n$ & {$[\mathrm{Ti} / \mathrm{Fe}] \mathrm{I}$} & $\mathrm{rms}$ & $n$ & {$[\mathrm{Ti} / \mathrm{Fe}] \mathrm{II}$} & $\mathrm{rms}$ \\
\hline 986 & 10 & +0.25 & 0.15 & 5 & +0.33 & 0.16 & 5 & +0.20 & 0.11 & & & \\
995 & 1 & +0.26 & & 6 & +0.32 & 0.23 & & & & & & \\
\hline
\end{tabular}

Table 6. Abundances of Fe-peak elements in stars of NGC 362. $n$ is the number of lines used in the analysis.

\begin{tabular}{|c|c|c|c|c|c|c|c|c|c|c|c|c|c|c|c|c|c|c|c|c|}
\hline Star & $n$ & {$[\mathrm{Sc} / \mathrm{Fe}] \mathrm{II}$} & $\mathrm{rms}$ & $n$ & {$[\mathrm{~V} / \mathrm{Fe}]$} & $\mathrm{rms}$ & $n$ & {$[\mathrm{Cr} / \mathrm{Fe}] \mathrm{I}$} & $\mathrm{rms}$ & $n$ & {$[\mathrm{Mn} / \mathrm{Fe}]$} & $\mathrm{rms}$ & $n$ & {$[\mathrm{Co} / \mathrm{Fe}]$} & $\mathrm{rms}$ & $n$ & {$[\mathrm{Ni} / \mathrm{Fe}]$} & $\mathrm{rms}$ & {$[\mathrm{Cu} / \mathrm{Fe}]$} & $\mathrm{rms}$ \\
\hline 986 & 5 & -0.10 & 0.10 & 6 & -0.05 & 0.08 & 5 & -0.03 & 0.14 & & & & 1 & -0.12 & & 12 & -0.07 & 0.11 & & \\
\hline 995 & 2 & -0.10 & 0.04 & 3 & -0.07 & 0.10 & 1 & -0.11 & & & & & & & & 3 & -0.16 & 0.09 & & \\
\hline
\end{tabular}

Table 7. Abundances of $n$-capture elements in stars of NGC 362 with UVES spectra; $n$ is the number of lines used in the analysis.

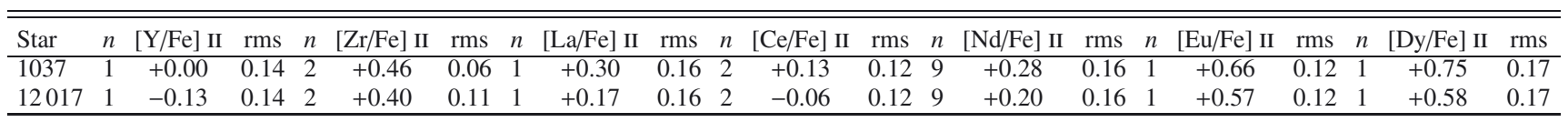

Notes. For species with only one transition, the scatter is estimated by summing in quadrature the best fit variation obtained by changing temperature, gravity, metal abundance and microturbulent velocity in amounts corresponding to the errors in these parameters. To this, we added a fit error of 0.05 dex in quadrature.

Table 8. Abundances of Ba II in stars of NGC 362. $n$ is the number of lines used in the analysis.

\begin{tabular}{cccc}
\hline \hline Star & $n$ & {$[\mathrm{Ba} / \mathrm{Fe}] \mathrm{II}$} & $\mathrm{rms}$ \\
\hline 986 & 1 & +0.400 & \\
995 & 1 & +0.447 & \\
\hline
\end{tabular}

the second one refers to the systematic effects. From the large sample of stars with GIRAFFE spectra, we derived a value of $[\mathrm{Fe} / \mathrm{H}]=-1.174 \pm 0.004 \pm 0.062 \mathrm{dex}(\mathrm{rms}=0.041 \mathrm{dex}, 90 \mathrm{stars})$. The metallicity of NGC 362 is very similar to that of NGC 1851 $([\mathrm{Fe} / \mathrm{H}]=-1.19$ dex, Carretta et al. 2011) and is 0.14 dex higher than the metal content of NGC $288([\mathrm{Fe} / \mathrm{H}]=-1.31 \mathrm{dex}$, Carretta et al. 2009c) on our homogeneous metallicity scale.

The abundances of iron obtained from singly ionised species agree with those from neutral lines: $[\mathrm{Fe} / \mathrm{H}] \mathrm{II}=-1.21$ $(\mathrm{rms}=0.08 \mathrm{dex}, 14$ stars $)$ from UVES and $[\mathrm{Fe} / \mathrm{H}] \mathrm{II}=-1.18$ $(\mathrm{rms}=0.06 \mathrm{dex}, 69 \mathrm{stars})$ from GIRAFFE. They do not present any trend as a function of the effective temperature, as shown in Fig. 4.

Our Fe abundance agrees very well with that listed in the old (1996) version of the Harris catalogue and with that obtained from analysis of the pulsational properties of RR Lyrae by Szekely et al. (2007). It is higher (although in agreement within the uncertainties) than the value listed in the most recent version of the Harris catalogue and the average values obtained by Shetrone \& Keane (2000) and Kraft \& Ivans (2003).

\section{Results}

\subsection{Chemistry of multiple populations in NGC 362}

The Na-O anti-correlation in NGC 362 is based on 71 stars for which we were able to provide $\mathrm{O}$ and $\mathrm{Na}$ abundances from UVES and/or GIRAFFE spectra. We have 58 detections and 13 upper limits for $\mathrm{O}$; the total number of stars with $[\mathrm{Na} / \mathrm{Fe}]$ values is 92. Results are shown in Fig. 5.

The interquartile range IQR for the ratio $[\mathrm{O} / \mathrm{Na}]$ in this cluster is 0.644 dex. This value nicely fits into the correlation with the total absolute magnitude of the cluster $\left(M_{V}=-8.41\right.$, Harris 1996) established in Carretta et al. (2010a). NGC 362 also participates to the relation with the maximum temperature along the HB ( $\log T_{\text {eff }}=4.079$, from Recio-Blanco et al. 2006), discovered by Carretta et al. (2007b) and repeatedly updated and strengthened during our FLAMES survey. NGC 362 stars seem to be clustered along the $\mathrm{Na}-\mathrm{O}$ anti-correlation in two distinct groups: one with high $\mathrm{O}$ and low $\mathrm{Na}$ and the other more enriched in $\mathrm{Na}$ and depleted in $\mathrm{O}$. This sub-division is even more evident if we consider only the 14 stars observed at higher resolution with UVES, and it is nicely supported by the distribution of the $[\mathrm{O} / \mathrm{Na}]$ ratios shown in Fig. 6 that maximise the signal along the 
Table 9. Mean abundances from UVES and GIRAFFE.

\begin{tabular}{lccc}
\hline \hline Element & \multicolumn{2}{c}{ UVES } & \multicolumn{2}{c}{ GIRAFFE } \\
& $n$ avg rms & $n$ avg rms \\
\hline$[\mathrm{O} / \mathrm{Fe}] \mathrm{I}$ & $14+0.140 .20$ & $64+0.890 .18$ \\
{$[\mathrm{Na} / \mathrm{Fe}] \mathrm{I}$} & $14+0.190 .19$ & $90+0.110 .25$ \\
{$[\mathrm{Mg} / \mathrm{Fe}] \mathrm{I}$} & $14+0.330 .04$ & $84+0.330 .04$ \\
{$[\mathrm{Al} / \mathrm{Fe}] \mathrm{I}$} & $14+0.240 .19$ & \\
{$[\mathrm{Si} / \mathrm{Fe}] \mathrm{I}$} & $14+0.220 .04$ & $87+0.260 .04$ \\
{$[\mathrm{Ca} / \mathrm{Fe}] \mathrm{I}$} & $14+0.300 .03$ & $89+0.340 .02$ \\
{$[\mathrm{Sc} / \mathrm{Fe}] \mathrm{II}$} & $14-0.030 .05$ & $90-0.070 .04$ \\
{$[\mathrm{Ti} / \mathrm{Fe}] \mathrm{I}$} & $14+0.220 .04$ & $84+0.160 .03$ \\
{$[\mathrm{Ti} / \mathrm{Fe}] \mathrm{II}$} & $14+0.210 .05$ & \\
{$[\mathrm{~V} / \mathrm{Fe}] \mathrm{I}$} & $14-0.030 .03$ & $86-0.050 .03$ \\
{$[\mathrm{Cr} / \mathrm{Fe}] \mathrm{I}$} & $14-0.020 .07$ & $88-0.030 .04$ \\
{$[\mathrm{Mn} / \mathrm{Fe}] \mathrm{I}$} & $14-0.330 .04$ & \\
{$[\mathrm{Fe} / \mathrm{H}] \mathrm{I}$} & $14-1.170 .05$ & $90-1.170 .04$ \\
{$[\mathrm{Fe} / \mathrm{H}] \mathrm{II}$} & $14-1.210 .08$ & $69-1.180 .06$ \\
{$[\mathrm{Co} / \mathrm{Fe}] \mathrm{I}$} & $14-0.280 .06$ & $36-0.050 .09$ \\
{$[\mathrm{Ni} / \mathrm{Fe}] \mathrm{I}$} & $14-0.130 .03$ & $90-0.090 .04$ \\
{$[\mathrm{Cu} / \mathrm{Fe}] \mathrm{I}$} & $14-0.500 .12$ & \\
{$[\mathrm{Zn} / \mathrm{Fe}] \mathrm{I}$} & $12+0.210 .06$ & $43+0.280 .08$ \\
{$[\mathrm{Y} / \mathrm{Fe}] \mathrm{II}$} & $14+0.070 .11$ & \\
{$[\mathrm{Zr} / \mathrm{Fe}] \mathrm{II}$} & $14+0.500 .12$ & \\
{$[\mathrm{Ba} / \mathrm{Fe}] \mathrm{II}$} & $14+0.300 .27$ & $68+0.180 .21$ \\
{$[\mathrm{La} / \mathrm{Fe}] \mathrm{II}$} & $14+0.330 .09$ & \\
{$[\mathrm{Ce} / \mathrm{Fe}] \mathrm{II}$} & $14+0.140 .12$ & \\
{$[\mathrm{Nd} / \mathrm{Fe}] \mathrm{II}$} & $14+0.350 .10$ & \\
{$[\mathrm{Eu} / \mathrm{Fe}] \mathrm{II}$} & $14+0.700 .07$ & \\
{$[\mathrm{Dy} / \mathrm{Fe}] \mathrm{II}$} & $14+0.680 .13$ & \\
\hline & &
\end{tabular}

$\mathrm{Na}-\mathrm{O}$ anti-correlation. We also note that the same separation into two groups was already present in the independent analysis by Shetrone \& Keane (2000, see their Fig. 2).

Using the quantitative criteria introduced by Carretta et al. (2009a), we can use $\mathrm{O}$ and $\mathrm{Na}$ abundances to quantify the size of the primordial $(\mathrm{P})$ population and of the intermediate (I) and extreme (E) components of the second generation stars. We found that the fractions of $\mathrm{P}, \mathrm{I}$, and E stars for NGC 362 are $22 \pm 6 \%$, $75 \pm 10 \%$, and $3 \pm 2 \%$, respectively. According to these criteria, we note that the lower- $\mathrm{Na} /$ high-O group visible in Fig. 5 includes not only the $\mathrm{P}$ component $([\mathrm{Na} / \mathrm{Fe}]<-0.03$ dex, in NGC 362) but also part of the intermediate fraction I. We warn however that the distribution of stars along the $\mathrm{Na}-\mathrm{O}$ anticorrelation from GIRAFFE spectra may be not optimally suited to study the discrete vs. continuous nature of multiple populations.

The radial distribution of these components is shown in Fig. 7, where the second generation stars of the I and E component are merged together, since only two stars belong to the E fraction. We find that second generation stars are more centrally concentrated, which is a result already found in several other clusters (see e.g. Lardo et al. 2011; Nataf et al. 2011; Milone et al. 2012).

The interplay of the various elements involved in protoncapture processes in NGC 362 is summarized in Fig. 8 for the stars observed with UVES. For this sub-sample, many light element abundances $(\mathrm{O}, \mathrm{Na}, \mathrm{Mg}, \mathrm{Al}, \mathrm{Si})$ are available from our data thanks to the large spectral coverage. As a reference, we also plot the results for 10 RGB stars observed with UVES in NGC 288 by Carretta et al. (2009b) in this figure. The range of Al variations is not extreme among giants in NGC 362, yet it is almost twice the spread in Al observed in NGC 288. The Al-O anti-correlation is well developed in NGC 362, whose O abundances reach a level significantly lower than in NGC 288, as found by Shetrone and Keane (2000). All these features can be explained by the higher

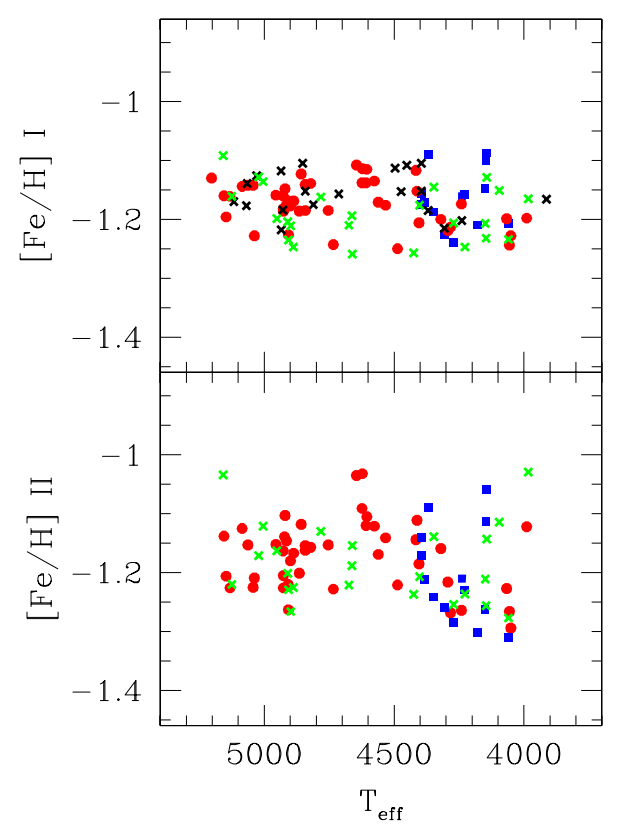

Fig. 4. Abundance ratios $[\mathrm{Fe} / \mathrm{H}]$ I (upper panel) and $[\mathrm{Fe} / \mathrm{H}]$ II (lower panel) as a function of $T_{\text {eff }}$ for all analysed stars. Blue squares are stars with UVES spectra; filled circles are those with GIRAFFE spectra, as observed with both HR11 and HR13 gratings, whereas crosses indicate stars observed with only the HR11 (black) or the HR13 (green) grating.

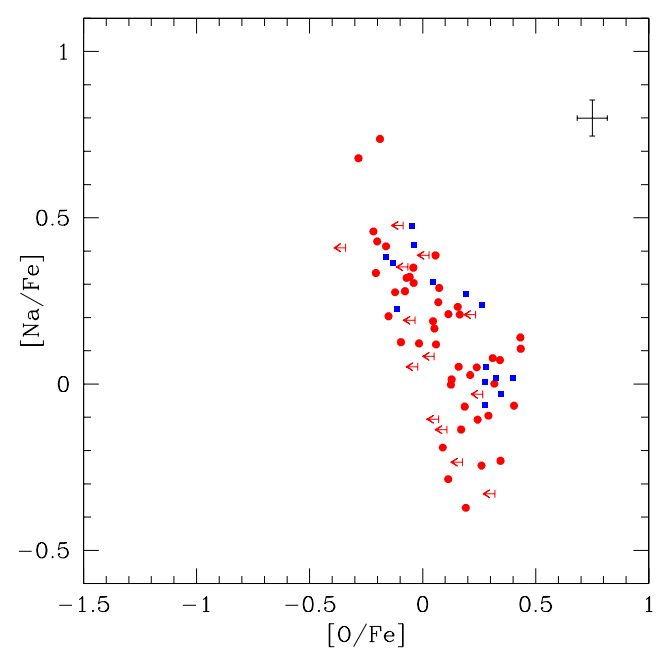

Fig. 5. Na-O anti-correlation observed in NGC 362. Blue squares are stars observed with UVES, while filled circles indicate stars with GIRAFFE spectra. Upper limits in $\mathrm{O}$ are shown as arrows, and starto-star (internal) error bars are plotted in the upper-right corner of the plot.

mass of NGC 362, which is almost five times more massive than NGC 288 (see McLaughlin \& van der Marel 2005).

Due to the limited size of the sub-sample of stars with UVES spectra, it is not possible to evince whether the polluting matter producing second generation stars was processed at very high temperature. As discussed by Yong et al. (2005) and Carretta et al. (2009b), a leakage from the $\mathrm{Mg}-\mathrm{Al}$ cycle on ${ }^{28} \mathrm{Si}$ occurs when the H-burning temperature exceeds $65 \mathrm{MK}$ and some amount of $\mathrm{Si}$ is produced at the expense of $\mathrm{Mg}$, in addition to the main outcome, Al. To verify that this is also the case for NGC 362, we show the run of $[\mathrm{Mg} / \mathrm{Fe}]$ and $[\mathrm{Si} / \mathrm{Fe}]$ ratios as a function of $\mathrm{O}$ and $\mathrm{Na}$ for the much larger sample of giants observed with GIRAFFE in this cluster in Fig. 9. 


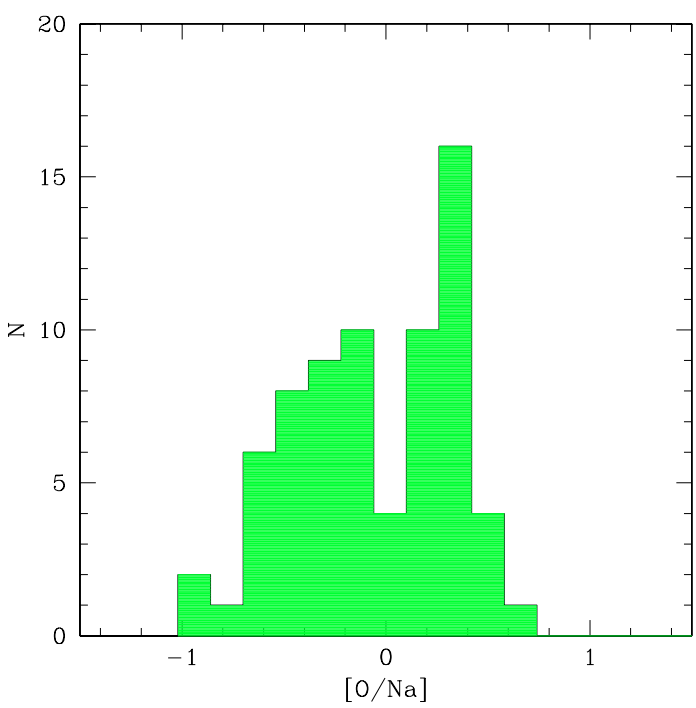

Fig. 6. Distribution of the $[\mathrm{O} / \mathrm{Na}]$ ratios in NGC 362 from the combined sample UVES+Giraffe.

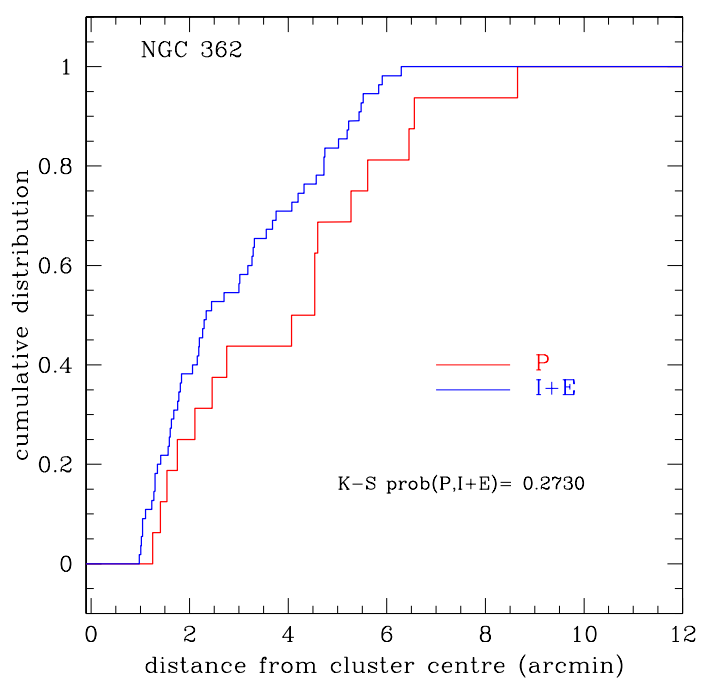

Fig. 7. Cumulative distribution of the stars of first (primordial P component) and second generation (the merged $\mathrm{I}+\mathrm{E}$ components together) as a function of the projected distance from the cluster centre.

$\mathrm{Mg}$ is correlated to elements depleted in proton-capture reactions, and anti-correlated to elements which are enhanced in this burning, and the opposite is seen to occur for Si. All these relations are statistically robust, the level of confidence formally exceeds $99 \%$ in all four cases. However, the relations involving $\mathrm{Si}$ seem to be driven by a few stars only. While the stars with UVES spectra give Mg abundances that nicely fall on the relations defined by the GIRAFFE sample involving $\mathrm{Mg}$, the agreement is not so satisfactory concerning $\mathrm{Si}$. In summary, it is not clear that the first generation polluters were of the right mass (inner temperature) range to significantly change the Si abundance above the level typical of enrichment from type II supernovae. In NGC 362, we did not observe the typical Si-Al correlation that suggests in massive or metal-poor GCs (such as NGC 2808 or NGC 6752, Carretta et al. 2009b) that Si is partly produced by proton-capture reactions, in addition to the classical $\alpha$-capture processes.
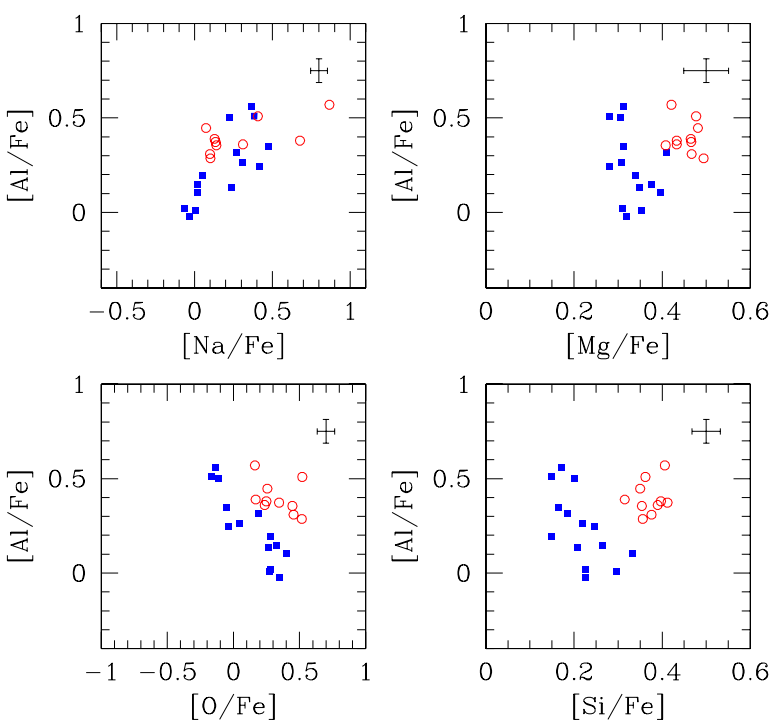

Fig. 8. Relations of the $[\mathrm{Al} / \mathrm{Fe}]$ ratios in NGC 362 (filled blue squares) from UVES spectra as a function of $[\mathrm{Na} / \mathrm{Fe}]$ (upper left panel), $[\mathrm{Mg} / \mathrm{Fe}]$ (upper right), $[\mathrm{O} / \mathrm{Fe}]$ (lower left panel), and $[\mathrm{Si} / \mathrm{Fe}]$ (lower right). The star to star error bars are indicated in each panel. As a comparison, the open circles are results for giants in NGC 288 from Carretta et al. (2009b).
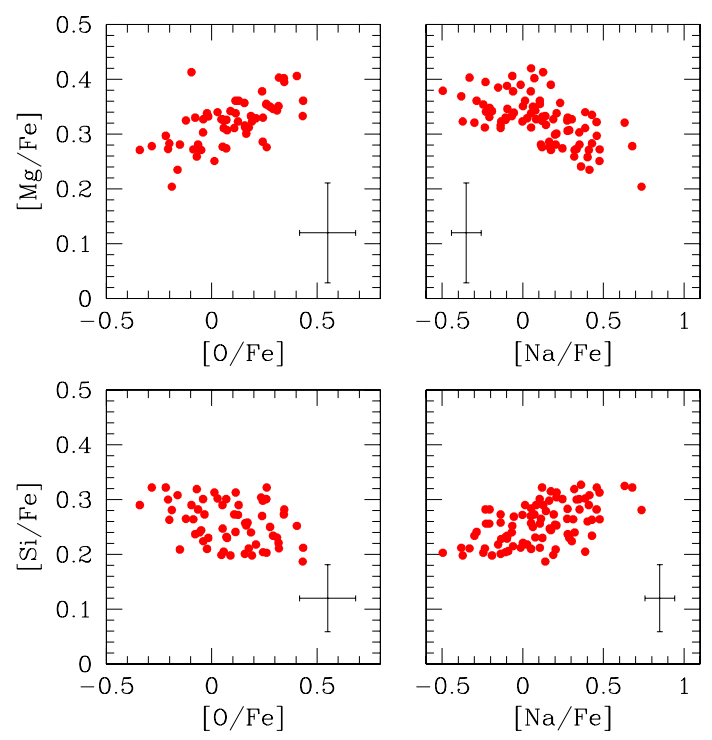

Fig. 9. $[\mathrm{Mg} / \mathrm{Fe}]$ ratios (upper panels) as a function of $[\mathrm{O} / \mathrm{Fe}]$ (left) and $[\mathrm{Na} / \mathrm{Fe}]($ right) ratios from GIRAFFE spectra. The same relations for $[\mathrm{Si} / \mathrm{Fe}]$ are plotted in the lower panels. The internal errors are indicated in each panel.

\subsection{Other elements}

The pattern of the $\alpha$ - and Fe-group element abundances is summarized in Figs. 10 and 11, where the abundances are plotted as a function of the effective temperature for individual stars in NGC 362.

In the last panel (bottom right) of Fig. 11, we also show the abundance ratios of $\mathrm{Ba}$, which is a neutron-capture element whose abundance in the solar system is mostly due to the $s$-process. None of these elements present a trend as a function of temperature, and, as shown in Table 9, their abundances are usually very homogeneous in NGC 362, apart from the light elements involved in proton-capture reactions that were discussed 

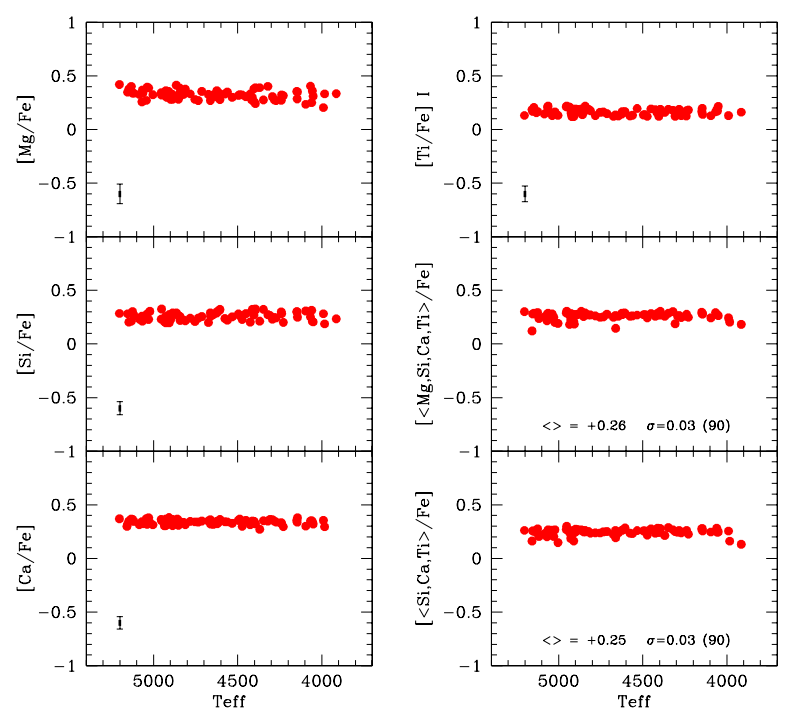

Fig. 10. Abundance ratios of $\alpha$-elements $\mathrm{Mg}, \mathrm{Si}, \mathrm{Ca}$, and $\mathrm{Ti}$ I as a function of the effective temperature. The average of $[\alpha / \mathrm{Fe}]$ ratios are shown in the last two panels on the right column (including and excluding the $\mathrm{Mg}$ abundance from the mean, respectively). Error bars indicate internal star-to-star errors.
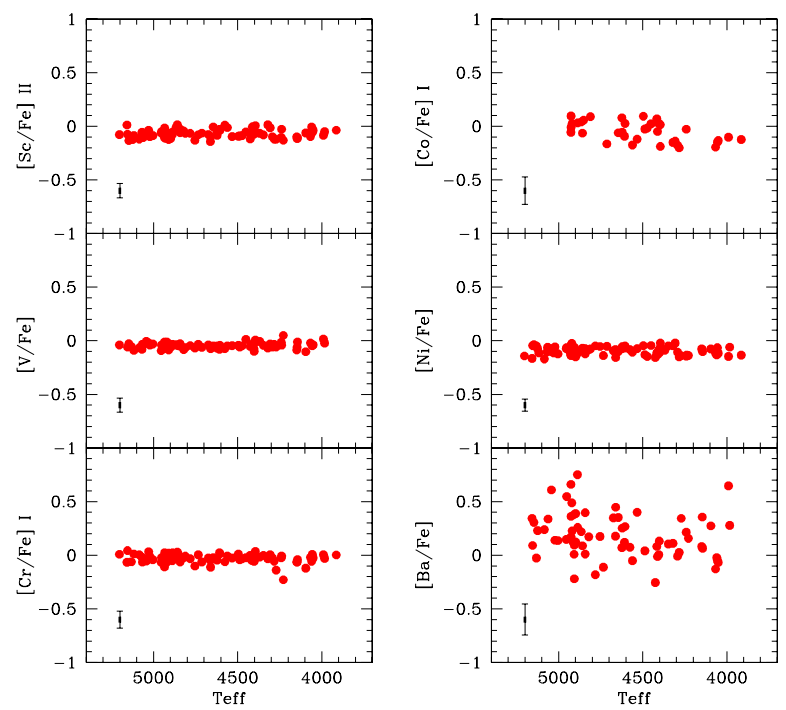

Fig. 11. Abundance ratios of elements of the Fe-peak (Sc II, V, Cr, Co, $\mathrm{Ni}$ ) and the $s$-process element $\mathrm{Ba}$ from GIRAFFE spectra as a function of the effective temperature. Error bars indicate internal star to star errors.

in the previous section. The case of Ba with its large spread are discussed in detail in Sect. 5.4 below.

Analysis of the UVES spectra allows the measurements of the abundances of several more $n$-capture elements, including $\mathrm{Cu}, \mathrm{Y}, \mathrm{La}, \mathrm{Ce}, \mathrm{Nd}, \mathrm{Eu}$, and $\mathrm{Dy}$, except for $\mathrm{Ba}$, which is also measured from GIRAFFE spectra. Since stars observed with UVES are typically very cool and luminous, some of the relevant lines are strong and then derived abundances are highly sensitive to microturbulence velocity. For some lines, we obtained a strong correlation between abundances and the adopted microturbulent velocities. As an example, Fig. 12 shows the correlations obtained for three clean Y II lines. The lines at 4883.7 and $5087.4 \AA$ show a marked trend, therefore we only used the line at $5200.4 \AA$. With similar considerations, we selected only Nd lines with EW smaller than $70 \mathrm{~m} \AA$, yielding typically six lines per

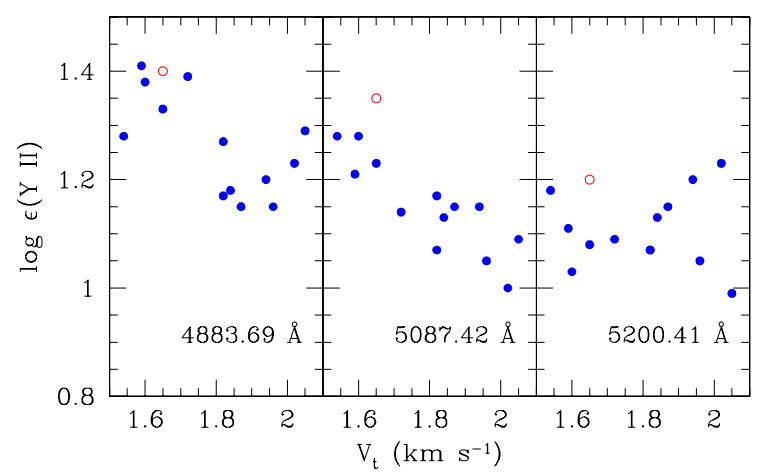

Fig. 12. $\log \epsilon$ (Y II) as derived from the three available clean lines in our spectral range as a function of micro-turbulent velocity. Star 11413 on the secondary, red RGB is indicated with a red open circle.

stars. In the case of $\mathrm{Ba}$, the three available lines, 5853.7, 6141.7, and $6469.9 \AA$ are all very strong, and thus, all sensitive to microturbulence. Figure 13 shows $[\mathrm{Zr} / \mathrm{Fe}],[\mathrm{Ba} / \mathrm{Fe}]$, and $[\mathrm{La} / \mathrm{Fe}]$ as a function of microturbulent velocity. The trend of Ba with microturbulence is quite obvious. However, part of the trend is due to star 11413 , which is richer in all n-capture elements than the other giants in the UVES sample. When this star is excluded, the trend between $\mathrm{Ba}$ abundances and microturbulence values is scarcely significant at less than two-sigma.

We note that the star-to-star variation in this parameter should be decreased to further flatten the trend of $\mathrm{Ba}$ (and of the strong Nd and Y lines) with microturbulence. That is, the low microturbulence values increased and the high ones decreased. However, this would create a trend of the Fe abundances with microturbulence of the opposite sign. Given that the measured Fe lines typically form deeper in the stellar atmospheres than the strong lines of heavy elements, this opposite behaviour with respect to microturbulence hints at an intrinsic inadequacy of the model atmosphere for cool and luminous stars. Note that this effect is likely not as strong in those stars observed using GIRAFFE (see Fig. 11), as they are typically warmer and fainter. Therefore, the derived errors for Ba indicated in Table A. 1 for the UVES stars should be taken with caution, as the true errors are likely larger, due to the very high sensitivity to the adopted microturbulent velocity. Finally, we note that an even stronger trend of $\mathrm{Ba}$ abundances with microturbulent velocity is present in the analysis by Shetrone \& Keane (2000), albeit Ba abundances increase as $V_{\mathrm{t}}$ increases in that case.

Figures 14-16 show the derived abundances for the heavy elements from UVES spectra as a function of $T_{\text {eff }}$. There is a weak trend of $\mathrm{Ba}$ abundances with $T_{\text {eff }}$ due to the above discussed correlation between derived $\mathrm{Ba}$ abundance and microturbulent velocity, given that the latter has a trend with effective temperature. Apart from the case of $\mathrm{Ba}$, the measured n-capture elements abundances are uniform, showing remarkably low scatter, especially when star 11413 is excluded from the average. This giant has the highest abundance of Y, Ba, La, Nd, Ce, and Dy among the sample of 14 stars with UVES spectra, whereas its Eu abundance does not stand out among the other stars in this sample.

\section{3. s- and r-process contributions}

The abundances of neutron capture elements derived from UVES spectra can be used to estimate the relative weight of the $s$ - and $r$-process contributions to the chemical pattern in NGC 362, as done in Carretta et al. (2011) for NGC 1851 and in 


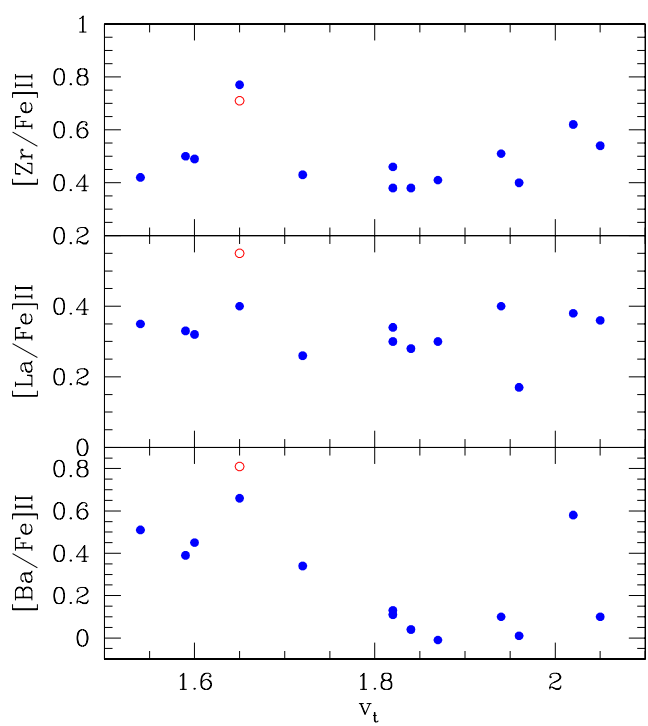

Fig. 13. Abundances of $\mathrm{Zr}, \mathrm{Ba}$ and $\mathrm{La}$ as a function of micro-turbulent velocity for stars with UVES spectra. The empty circle indicates star 11413 (see text)

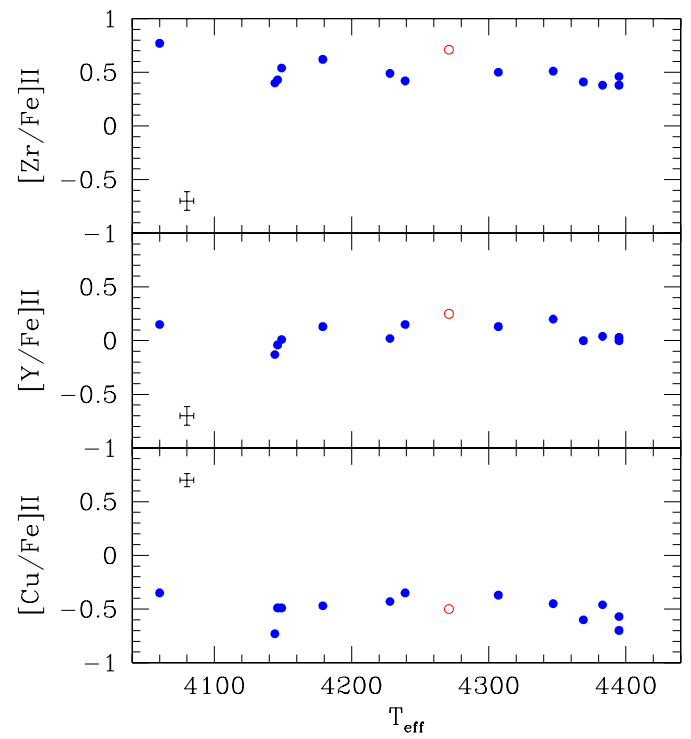

Fig. 14. Abundance ratios of elements of the $n$-capture elements $\mathrm{Zr}$ II, $\mathrm{Y}$ II, and $\mathrm{Cu}$ I, from UVES spectra as a function of the effective temperature. Error bars indicate internal star-to-star errors. In each panel, the Ba-rich star 11413 is highlighted.

D'Orazi et al. (2011) for $\omega$ Cen. We refer to those papers for the details on the adopted procedure. Results for $\mathrm{Ba}, \mathrm{La}, \mathrm{Ce}$, and $\mathrm{Eu}$ are well consistent with each other: the pattern of abundances is reproduced if we assume that most of these elements are made by the $r$-process ( $1 / 3$ of the solar $r$-process abundances) with a small contribution from the $s$-process (about $1 / 25$ of the solar $s$-process abundances).

On the other hand, results for the first-peak elements, Y and $\mathrm{Zr}$, are discrepant: we found less $\mathrm{Y}$ and too much $\mathrm{Zr}$ with respect to the above recipe. The uncertainties in the determination of $\mathrm{Y}$ and $\mathrm{Zr}$ do not allow to determine with precision the pattern of the first peak species. Since we have well defined abundances only for the second peak, we are unable to calculate the ratio between heavy and light $s$-process elements in NGC 362. Hence, nothing more can be said about the origin of

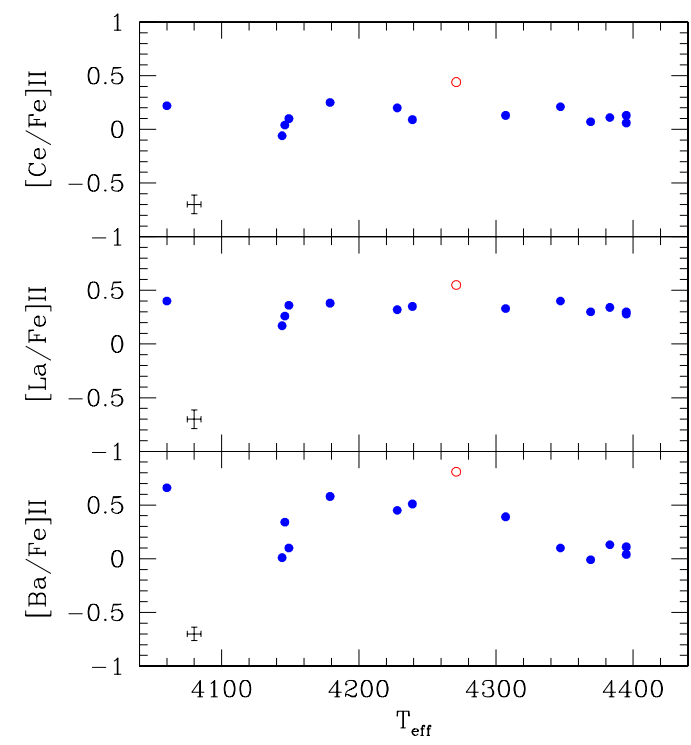

Fig. 15. As in Fig. 14 for the $n$-capture elements Ba II, La II, and Ce II.

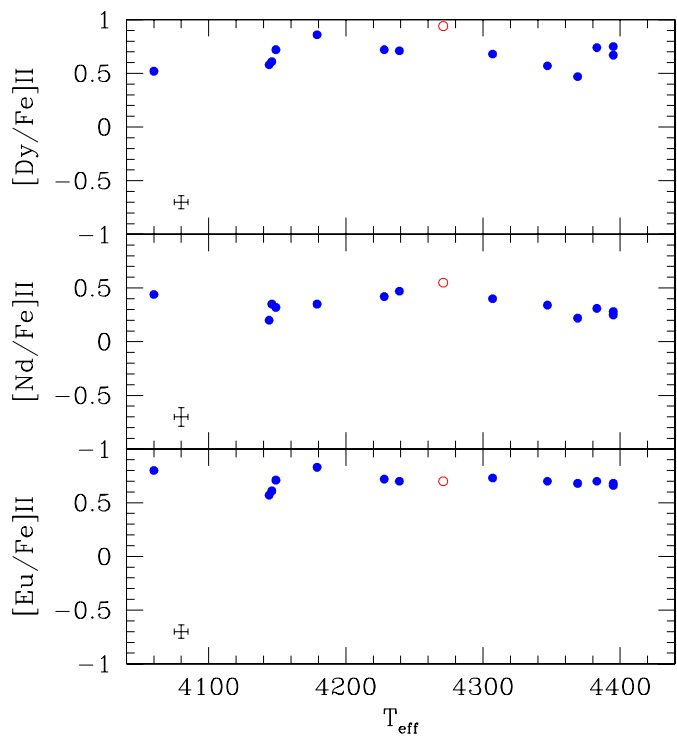

Fig. 16. As in Fig. 14 for the $n$-capture elements Eu II, Nd II, and Dy II.

these elements; in turn, the timescales of the enrichment cannot be properly determined.

\subsection{Ba and the red sequence on the RGB}

The Ba abundances for about 70 stars observed with GIRAFFE are based on a single line at $6141 \AA$, and the associated internal error is large $(\sim 0.15 \mathrm{dex})$. However, the rms scatter of the mean $(0.21 \mathrm{dex})$ is greater than the internal errors (see Fig. 11, bottom right panel, and Fig. 15). Additional evidence that the spread may be real, albeit small, comes from the Strömgren photometry. We used the photometry collected by Grundahl and coworkers and presented by Calamida et al. $(2007)^{7}$ to whom we refer for details. There are 69 stars having both photometric data and abundances of $\mathrm{Ba}$. We show the Strömgren $y, v-y$ CMD for these stars in Fig. 17, where we indicate stars with $\mathrm{Ba}$ abundances that are lower or higher than the average value of

7 The catalogue was downloaded from the web page http://www.oa-roma.inaf.it/spress/gclusters.html 


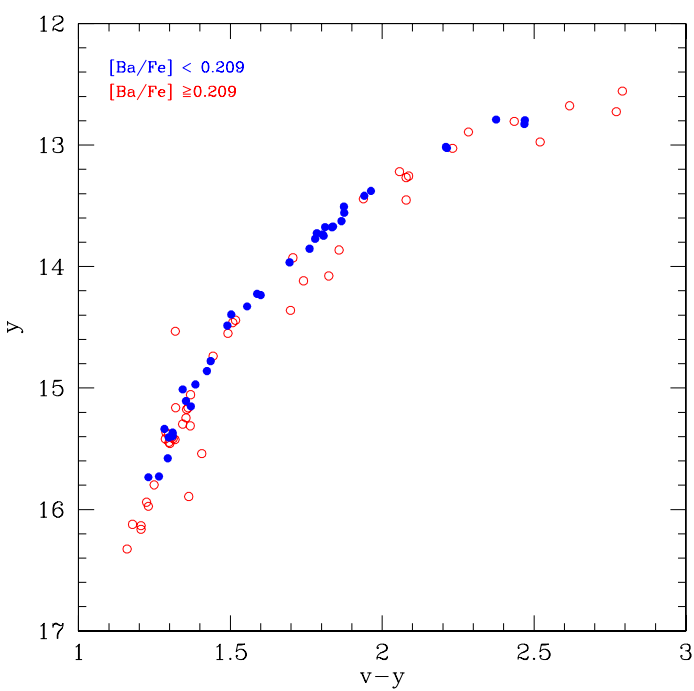

Fig. 17. Strömgren $y, v-y$ CMD for stars of our sample with accurate photometry. The stars are divided according to their $\mathrm{Ba}$ abundances. Blue filled circles are stars with lower $[\mathrm{Ba} / \mathrm{Fe}]$ than the average value $[\mathrm{Ba} / \mathrm{Fe}]=+0.209$ dex, whereas red open circles indicate stars with $\mathrm{Ba}$ content larger than the mean value.

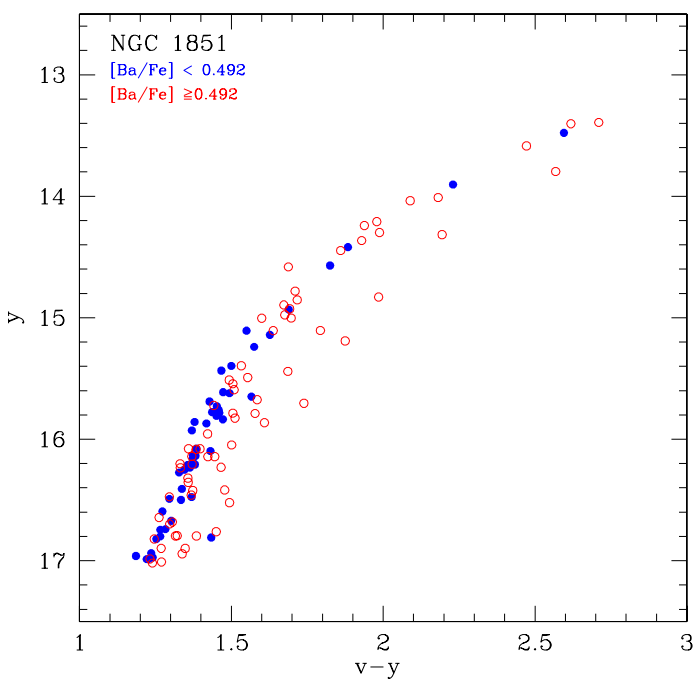

Fig. 18. Strömgren $y, v-y$ CMD for stars of NGC 1851 from Carretta et al. (2011). The stars are divided according to their $\mathrm{Ba}$ abundances, blue filled circles and red open circles being stars with lower and higher $[\mathrm{Ba} / \mathrm{Fe}]$, respectively, than the average value $[\mathrm{Ba} / \mathrm{Fe}]=+0.492 \mathrm{dex}$.

$[\mathrm{Ba} / \mathrm{Fe}]=+0.209$ dex with different symbols. Stars with lower than average $\mathrm{Ba}$ abundances define a very narrow sequence at the blue ridge of the RGB, while a fraction of the Ba-rich stars populate a sequence slightly to the red of the main RGB (apart from an outlier with a very blue $v-y$ colour that is likely due to errors in the photometry). We come back to this red sequence in Sect. 6

We note that the above discussed uncertainty in the Ba abundance due to microturbulence does not affect this result. At a given effective temperature $T_{\text {eff }}$, the relative $\mathrm{Ba}$ abundances are robust with respect to microturbulence; therefore the separation between $\mathrm{Ba}-$ rich and $\mathrm{Ba}-$ poor stars at a given magnitude is reliable.

This behaviour reproduces the splitting of the RGB of NGC 1851 observed in the $v-y$ colours, as shown in Fig. 18,

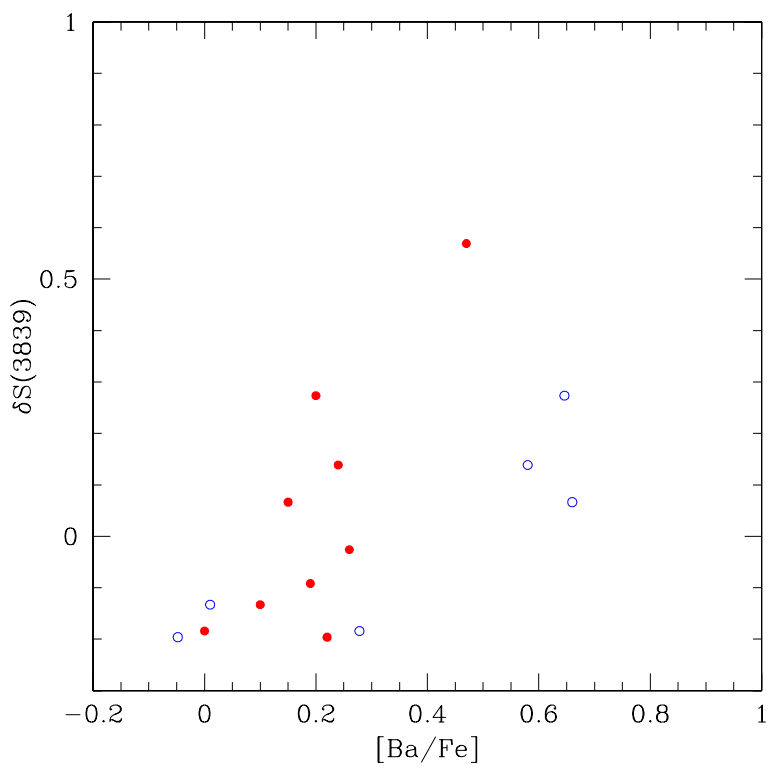

Fig. 19. Index $\delta S(3839)$ of the $\mathrm{CN}$ bandstrength from Smith \& Langland-Shula (2009) as a function of the $[\mathrm{Ba} / \mathrm{Fe}]$ ratio from Shetrone $\&$ Keane (2000, red filled point) and from the present work (blue open circles) for stars of NGC 362.

using data from Carretta et al. (2011). In both cases, the reddest sequence is populated entirely by Ba-rich stars.

What is the physical explanation for this phenomenon? In Carretta et al. (2011), we suggested that the redder $v-y$ colour of this secondary sequence is most likely due to large enhancements in N. Very recently, this suggestion was supported by the analysis of $\mathrm{N}$ abundances based on a number of $\mathrm{CN}$ features measured on GIRAFFE spectra (Carretta et al., in prep.), and on a limited sample of giants by Villanova et al. (2010) in NGC 1851. The stars populating the reddest sequence all have strong $\mathrm{CN}$ bands and higher $\mathrm{N}$ abundances.

The low resolution data for bright giants by Smith \& Langland-Shula (2009) may be used to check if the same holds for NGC 362. These authors provided values of the index $S$ (3839) which measure the strength of the $\mathrm{CN}$ band strength. These values were converted into the quantity $\delta S$ (3839) as defined by Norris (1981) using for the baseline the equation $S(3839)=0.44+0.0878 \times M_{V}$ based on the data in Smith \& Langland-Shula (2009). Note that the stars in common between the two samples span a very limited range in magnitude and colour along the RGB of NGC $362\left(-2.23<M_{V}<-1.92\right)$. Hence, any variation in the $\delta S(3839)$ index with luminosity and/or temperature has no impact in our discussion.

We found that this index is also well correlated with the $[\mathrm{Ba} / \mathrm{Fe}]$ ratio, in addition to the already known correlation with $\mathrm{Na}$ and anti-correlation with $\mathrm{O}$ abundances. We notice that the same effect was found in M 22 by Marino et al. (2011). This is shown in Fig. 19, where we adopted Ba abundances from both the few stars of the present study and the more numerous giants in common with Shetrone and Keane (2000). Among the six stars in our sample, star 2333 with the highest value of $\delta S$ (3839) lies on the red RGB sequence.

Since $\mathrm{Ba}$ is correlated with $\mathrm{Na}$ and anti-correlated with $\mathrm{O}$ in this subset of stars, we checked that the same also holds in our much larger sample observed with GIRAFFE. In Figs. 20 and 21, we show the run of $\mathrm{Ba}$ abundances as a function of the content of $\mathrm{O}, \mathrm{Na}, \mathrm{Mg}$, and $\mathrm{Si}$. The best (anti-)correlations are those between $\mathrm{Ba}$ and the elements that are depleted in proton-capture 

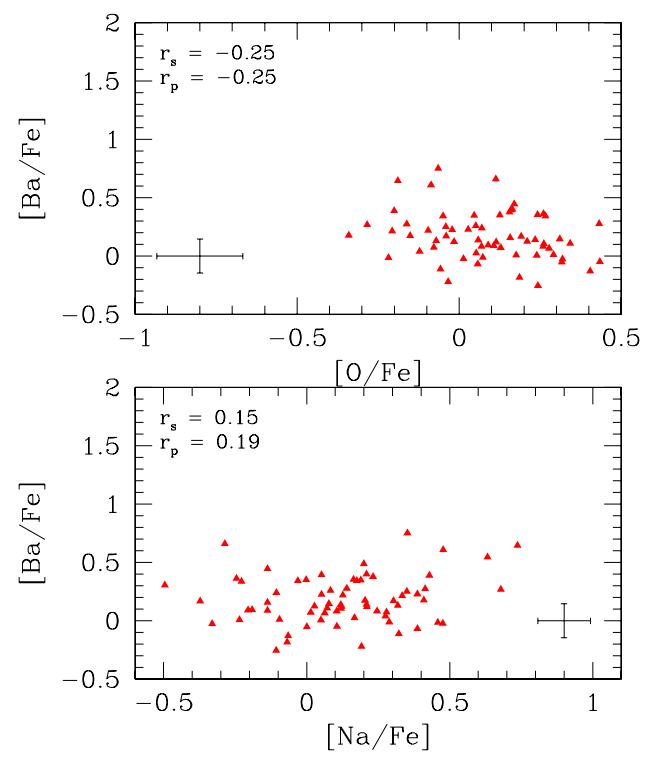

Fig. 20. Upper panel: $[\mathrm{Ba} / \mathrm{Fe}]$ as a function of $[\mathrm{O} / \mathrm{Fe}]$ in giants of NGC 362 from this study. Lower panel: $[\mathrm{Ba} / \mathrm{Fe}]$ as a function of $[\mathrm{Na} / \mathrm{Fe}]$. The Pearson linear correlation coefficient and the Spearman rank correlation coefficient with star to star error bars are indicated in each panel.

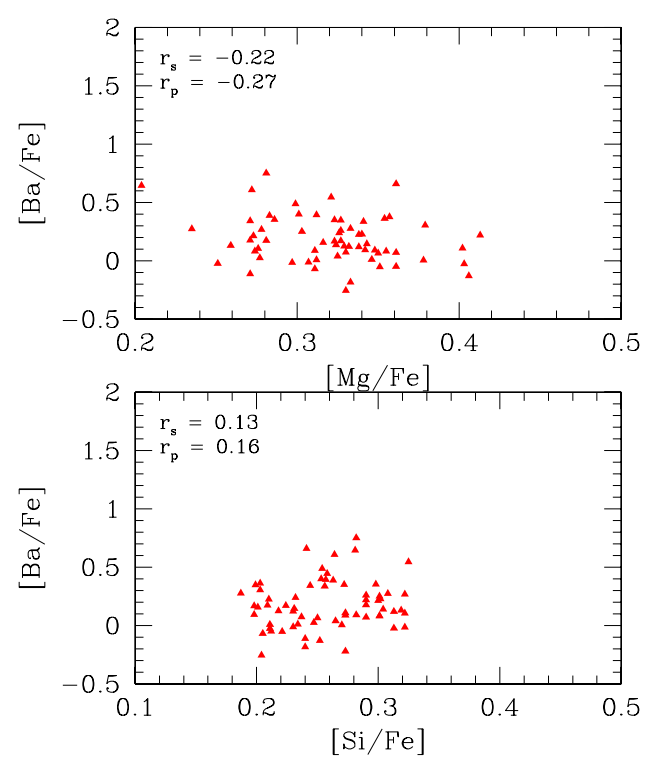

Fig. 21. As in Fig. 20 but for $[\mathrm{Mg} / \mathrm{Fe}]$ (upper panel) and $[\mathrm{Si} / \mathrm{Fe}]$ (lower panel).

reactions like $\mathrm{O}$ and $\mathrm{Mg}$. The linear correlation coefficients are $r=-0.25$ and -0.27 , respectively (with 61 and 62 degrees of freedom, respectively). These anti-correlations, although not striking, are significant to a level of confidence of about $95 \%$, admittedly not very high, while the complementary correlations with $\mathrm{Na}$ and $\mathrm{Si}$ are not statistically significant.

At the moment, the reason why a typical $s$-process element like $\mathrm{Ba}$ must be related to the abundances of elements forged in the nuclear processing of likely much more massive stars of the first stellar generation in the cluster still remains an unsolved issue, owing to the much different lifetimes of typical polluters for these elements. More efforts should be focused in the future in ascertaining the ground of these possible correlations in large samples of stars.

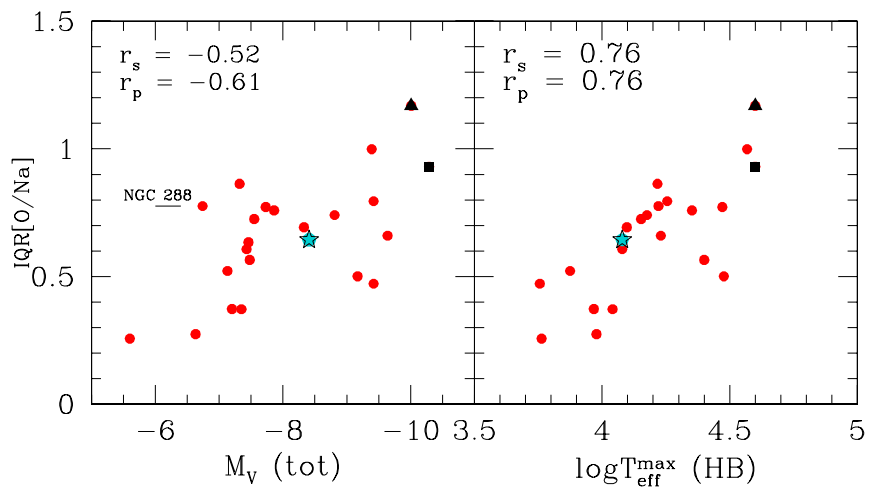

Fig. 22. Interquartile range (IQR) of the $[\mathrm{O} / \mathrm{Na}]$ ratio in $\mathrm{NGC} 362$ as a function of the total absolute magnitude of the cluster (left panel) and of the maximum temperature along the HB (right panel). NGC 362 is represented by a filled star symbol. The filled square is for $\omega$ Cen from Johnson \& Pilachowski (2010) and the filled triangle indicates M 54 (Carretta et al. 2010c). The other clusters are from Carretta et al. (2009a, 2011). In the left panel, the location of NGC 288 is indicated. In each panel, the Spearman rank correlation coefficient $\left(r_{\mathrm{s}}\right)$ and the Pearson's correlation coefficient are reported.

\section{Discussion and conclusions}

We studied the chemical composition of 92 red giant branch stars in the globular cluster NGC 362.

We found that NGC 362 behaves like a perfectly "normal" cluster for what concerns the elements involved in high temperature $\mathrm{H}$-burning. It nicely falls in the middle of the relation between the extension of the $\mathrm{Na}-\mathrm{O}$ anti-correlation and the total absolute magnitude (a proxy for the present-day cluster mass) defined by the other GCs (Carretta et al. 2010a; see left panel of Fig. 22). The same holds for the correlation of the extension of $\mathrm{Na}-\mathrm{O}$ anti-correlation with the extent of the $\mathrm{HB}$ distribution (right panel of Fig. 22).

In NGC 362, there is a hint that stars are clustered into two discrete groups along the $\mathrm{Na}-\mathrm{O}$ anti-correlation. This comes from both the more conspicuous sample of RGB stars with GIRAFFE spectra and the more limited fraction of stars with high-resolution FLAMES/UVES spectra.

First-generation stars (Na-poor and O-rich) are apparently less concentrated than second generation stars, in agreement with the result found in most GCs both from spectroscopy (e.g. Carretta et al. 2010d) and photometry (e.g. Lardo et al. 2011; Milone et al. 2012, and references therein); however, this finding is not supported by a high level of statistical confidence in NGC 362.

In this context, it is interesting to compare NGC 362 to its twin second-parameter cluster NGC 288 (Shetrone \& Keane 2000; and, in particular, Carretta et al. 2009a,b). The latter GC is indicated in Fig. 22 (left panel) to show its position slightly off the main relation. Carretta et al. (2010a) already discussed evidence (like tidal tails) pointing toward a large loss of stars for NGC 288 in the past. More evidence in this sense is given by the study by Paust et al. (2010), who determined the global present day mass functions for 17 GCs, including NGC 288 and NGC 362. Their comparison shows that NGC 288 has a flatter mass function (the slope of the power law fit is $\alpha=-0.83$ as compared to $\alpha=-1.69$ of NGC 362), indicating a lower fraction of low mass stars. Since NGC 288 is a more loose cluster than its twin NGC 362, this seems to imply that is also more affected by external processes of evaporation through disk shocking and/or 
tidal stripping (see Paust et al. 2010), explaining its position well in the plane IQR $[\mathrm{O} / \mathrm{Na}]$ vs. $M_{V}$.

Also relevant in the context of multiple populations in GCs is the recent addition of NGC 362 to the increasing number of clusters where a broad and/or even split subgiant branch (SGB) was detected. The studies by Milone and collaborators include: NGC 1851 (Milone et al. 2008), 47 Tuc (Anderson et al. 2009; Milone et al. 2012), NGC 6388 and (maybe) NGC 6441 (Bellini et al. 2013), NGC 362, NGC 5286, NGC 6656, NGC 6715, and NGC 7089 (Piotto et al. 2012).

Our FLAMES survey, devoted to studying the abundances of proton-capture elements (mainly $\mathrm{Na}$ and $\mathrm{O}$ ) in large samples of RGB stars in a large number of GCs, is blind to the feature of double SGBs, which is governed by age and/or CNO content (see e.g. Cassisi et al. 2008). However, a further peculiarity of NGC 362 is its secondary giant branch in CMD using the $v-y$ colour (see Fig. 17), as already noted in previous section.

The reality of the sequence and the membership of this population to the cluster is well assessed in the present work. Our spectroscopic analysis shows that all the stars on this secondary giant branch have measured RV and metallicity consistent with the cluster. This sequence is neither grossly contaminated by neither Galactic field interlopers nor stars of the Small Magellanic Cloud, found at fainter magnitudes and well separated from the stars in NGC 362 in the $y, v-y$ plane. Beside the split SGB, NGC 362 thus seems to be part of the group of GCs where a secondary giant branch is found in the $y, v-y$ plane. The most relevant cases include NGC 1851, NGC 6656 (M 22), and NGC 7089 (M 2: Lardo et al. 2012). With the addition of NGC 362, the phenomenon becomes common enough to justify the dubbing of secondary RGB for this feature, since it seems to enclose only a minor fraction of the total red giants, although the percentage does vary from cluster to cluster.

In the present study, we showed that the secondary RGB of NGC 362 is only populated by stars with high Ba abundance, compared to the average of the sample ${ }^{8}$. The conspicuous blue RGB instead includes both Ba-rich and Ba-normal stars. Is this separation only restricted to the Ba abundances? Star 11413 is the only star on this sequence observed with UVES. Unfortunately, this star was classified as binary by F93. This would have no impact on the abundance analysis unless the binary system underwent mass transfer from an AGB companion, which is a rare event. While this occurrence would explain the kinematics and part of the chemical pattern of this star, it is surely not the explanation of the secondary sequence: if the stars on this sequence were this kind of binaries, we would expect a range of variations (also in colour) not a sequence. To be conservative, we cannot infer that the enhancement observed for $\mathrm{Ba}$ is extended to all the $s$-process elements from this star alone.

With the red sequence only populated by stars with high Ba abundances this pattern is reminiscent of the chemical characterization of the red RGB in NGC 1851 that are found to be preferentially enriched in $s$-process elements (and in particular, Ba) in a number of recent studies (Yong \& Grundahl 2008; Villanova et al. 2010; Carretta et al. 2011) ${ }^{9}$. Even more marked, the same pattern is present in M 22, where stars on the bluer RGB are however all $s$-poor and stars on the red RGB are all $s$-rich, as

\footnotetext{
8 It also seems to show a tendency for lower $\mathrm{O}$ and higher $\mathrm{Na}$ abundances, although we measured both elements only in six stars. This will be discussed more in a companion paper.

9 The small sample by Yong and Grundahl is not assigned to one of the RGBs; however, three s-rich stars are seen to lie on the reddest branch in Fig. 1 of Lee et al. (2009).
}
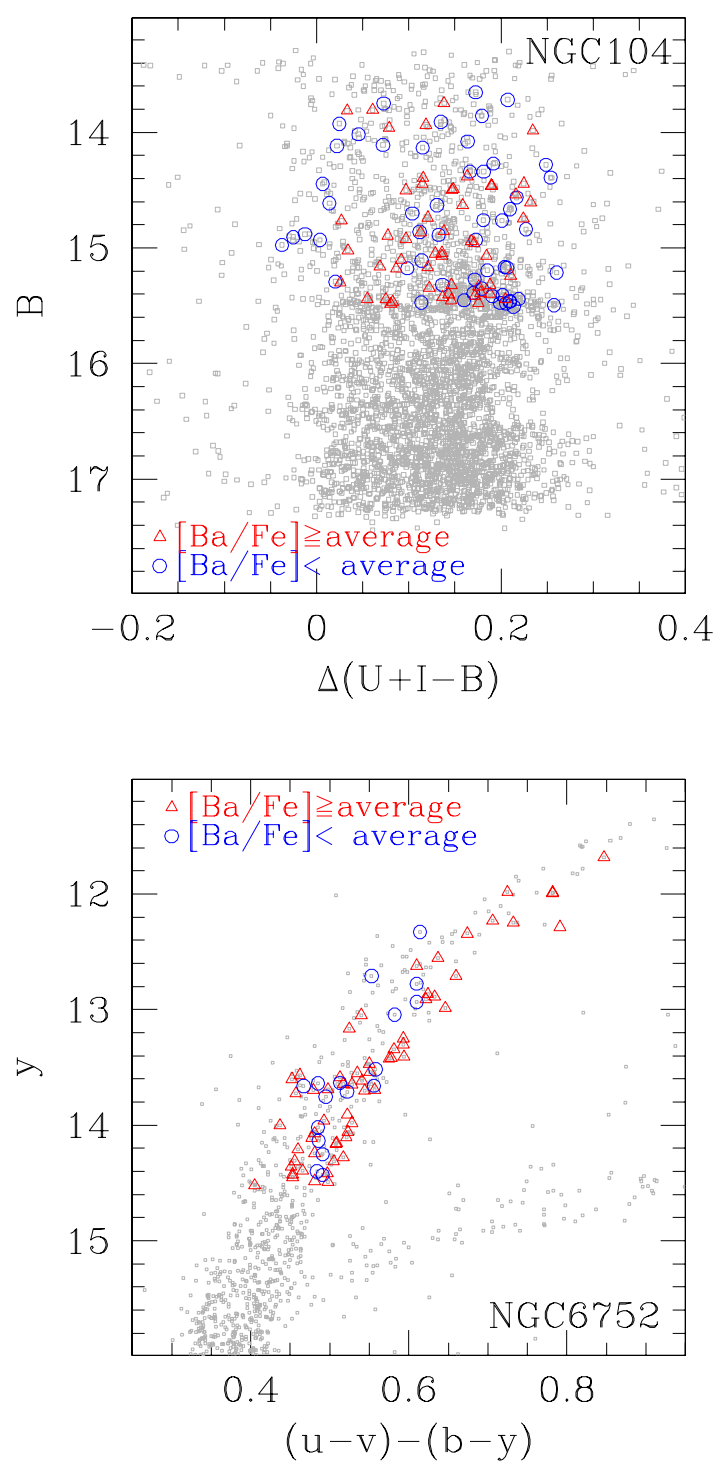

Fig. 23. Plot for 47 Tuc (top), using the same filter combination adopted in Milone et al. (2012) to show a split RGB (see their Figs. 21 and 26). The photometry is not the same. Ba-rich and Ba-poor stars are not segregated. For NGC 6752 (bottom), we show the same effect on a plot similar to the one in Milone et al. (2013; their Fig. 15).

demonstrated by Marino et al. $(2009,2011)$. In that cluster, the separation is more neat.

A more detailed discussion of the properties of this "secondary RGB" in NGC 362 and a comparison with other peculiar GCs will be presented in a separate paper (Carretta et al., in prep.). Here we only note that the splitting in the $y, v-y$ diagram seems to be different from the split RGB found in clusters, such as NGC 104 (47 Tuc) or NGC 6752, when using colours including ultraviolet magnitudes, which are closely related to the $\mathrm{Na}-\mathrm{O}$ anti-correlation (see the extensive photometric studies by Milone et al. 2012, 2013, and the theoretical interpretation by Sbordone et al. 2011). Not all splits in the different filter combinations have the same meaning (see the discussion in Carretta et al., in prep.; and Milone et al. 2012.). Using the photometry from Calamida et al. (2007) no secondary RGB is easily discernable for the two GCs just mentioned in the $y, v-y$ diagrams, which show that this feature is present in some, but not all GCs. These GCs also behave differently for what concerns Ba abundances. To show this, we used the Johnson UBVI photometry by 
Momany (unpublished photometry, reduced as in Momany et al. 2003) for 47 Tuc and the Strömgren photometry by Grundahl for NGC 6752 (Calamida et al. 2007) to reproduce the clear splits shown by Milone et al. $(2012,2013)$. We took the Ba abundances from D'Orazi et al. (2010), cross-identified the stars, and plotted the ones in common in Fig. 23. When we divide the sample in Ba-rich or Ba-poor stars with respect to the average $[\mathrm{Ba} / \mathrm{Fe}]$ value in each cluster (as done in NGC 1851 by Carretta et al. 2011 and in the present work for NGC 362), no segregation is evident on the split RGBs in these two clusters, which is at odds with what is seen for $\mathrm{C}, \mathrm{N}$ and $\mathrm{Na}, \mathrm{O}$ (Milone et al. 2012, 2013).

In the past years, we are learning new things on the nature of GCs, which are more complex and more interesting than we thought for long time. True progress and understanding requires a multi-lateral approach, making the best of the information that photometry, spectroscopy, and theoretical modelling can give us.

Acknowledgements. V.D. is an ARC Super Science Fellow. This publication makes use of data products from the Two Micron All Sky Survey, which is a joint project of the University of Massachusetts and the Infrared Processing and Analysis Center/California Institute of Technology, funded by the National Aeronautics and Space Administration and the National Science Foundation. This research has been funded by PRIN INAF 2011 "Multiple populations in globular clusters: their role in the Galaxy assembly" (PI E. Carretta), and PRIN MIUR 2010-2011, project "The Chemical and Dynamical Evolution of the Milky Way and Local Group Galaxies" (PI F. Matteucci). This research has made use of the SIMBAD database, operated at CDS, Strasbourg, France and of NASA's Astrophysical Data System.

\section{References}

Alonso, A., Arribas, S., \& Martinez-Roger, C. 1999, A\&AS, 140, 261 Alonso, A., Arribas, S., \& Martinez-Roger, C. 2001, A\&A, 376, 1039 Anderson, J., Piotto, G., King, I. R., Bedin, L., \& Guhathakurta, P. 2009, ApJ, 697, L58

Bellazzini, M., Fusi Pecci, F., Ferraro, F. R., et al. 2001, AJ, 122, 2569

Bellazzini, M., Bragaglia, A., Carretta, E., et al. 2012, A\&A, 538, A18

Bellini, A., Piotto, G., Milone, A. P., et al. 2013, ApJ, 765, 32

Boyer, M. L., McDonald, I., van Loon, J. Th., et al. 2009, ApJ, 705, 746

Calamida, A., Bono, G., Stetson, P. B., et al. 2007, ApJ, 670, 400

Carretta, E., Bragaglia, A., Gratton R. G., et al. 2006, A\&A, 450, 523

Carretta, E., Bragaglia, A., Gratton, R. G., et al. 2007a, A\&A, 464, 967

Carretta, E., Recio-Blanco, A., Gratton, R. G., Piotto, G., \& Bragaglia, A. 2007b, ApJ, 671, L125

Carretta, E., Bragaglia, A., Gratton, R. G., et al. 2009a, A\&A, 505, 117

Carretta, E., Bragaglia, A., Gratton, R. G., \& Lucatello, S. 2009b, A\&A, 505, 139

Carretta, E., Bragaglia, A., Gratton, R. G., D’Orazi, V., \& Lucatello, S. 2009c, A\&A, 508, 695

Carretta, E., Bragaglia, A., Gratton, R. G., et al. 2010a, A\&A, 516, A55

Carretta, E., Gratton, R. G., Lucatello, S., et al. 2010b, ApJ, 722, L1

Carretta, E., Bragaglia, A., Gratton, R. G., et al. 2010c, A\&A, 520, A95

Carretta, E., Bragaglia, A., D’Orazi, V., Lucatello, S., Gratton, R. G. 2010d, A\&A, 519, A71

Carretta, E., Lucatello, S., Gratton, R. G., Bragaglia, A., \& D’Orazi, V. 2011, A\&A, 533, A69

Cassisi, S., Salaris, M., Pietrinferni, A., et al. 2008, ApJ, 672, 115
Catelan, M., Bellazzini, M., Landsman, W. B., et al. 2001, AJ, 122, 3171

Clement, C. M. 1997, VizieR On-line Data Catalogue V/97, original catalog published in Sawyer Hogg. H. 1973, PDDO, 3, 6

Dinescu, D. I., Girard, T. M., \& van Altena, W. F. 1999, AJ, 117, 1792

D'Orazi, V., Gratton, R. G., Lucatello, S., et al. 2010, ApJ, 719, L213

D’Orazi, V., Gratton, R. G., Pancino, E., et al. 2011, A\&A, 534, A29

Dotter, A., Sarajedini, A., Anderson, J., et al. 2010, ApJ, 708, 698

Fischer, P., Welch, D. L., Mateo, M., \& Cote, P. 1993, AJ, 106, 1508

Gratton, R. G., Carretta, E., Eriksson, K., \& Gustafsson, B. 1999, A\&A, 350 955

Gratton, R. G., Bonifacio, P., Bragaglia, A., et al. 2001, A\&A, 369, 87

Gratton, R. G., Carretta, E., Claudi, R., Lucatello, S., \& Barbieri, M. 2003, A\&A, 404, 187

Gratton, R. G., Sneden, C., \& Carretta, E. 2004, ARA\&A, 42, 385

Gratton, R. G., Carretta, E., Bragaglia, A., Lucatello, S., \& D’Orazi, V. 2010, A\&A, 517, A81

Gratton, R. G., Carretta, E., \& Bragaglia, A. 2012, A\&ARv, 20, 50

Harris, W. E. 1996, AJ, 112, 1487

Johnson, C. I., \& Pilachowski, C. A. 2010, ApJ, 722, 1373

Kayser, A., Hilker, M., Grebel, E. K., \& Willemsen, P. G. 2008, A\&A, 486, 437

King, I. R. 1966, AJ, 71, 276

Kraft, R. P., \& Ivans, I. 2003, PASP, 115, 143

Kurucz, R. L. 1993, CD-ROM 13, Smithson. Astrophys.

Lardo, C., Bellazzini, M., Pancino, E., et al. 2011, A\&A, 525, A114

Lardo, C., Pancino, E., Mucciarelli, A., \& Milone, A. P. 2012, A\&A, 548, A107

Lee, J.-W., Lee, J., Kang, Y.-W., et al. 2009, ApJ, 695, L78

Magain, P. 1984, A\&A, 134, 189

Mackey A. D., \& van den Bergh, S. 2008, MNRAS, 360, 631

Marino, A. F., Villanova, S., Piotto, G., et al. 2008, A\&A, 490, 625

Marino, A. F., Milone, A., Piotto, G., et al. 2009, A\&A, 505, 1099

Marino, A. F., Sneden, C., Kraft, R. P., et al. 2011, A\&A, 532, A8

Martell, S. L. 2011, AN, 332, 467

Martell, S. L., Smolinski, J. P., Beers, T. C., \& Grebel, E. K. 2011, A\&A, 534, A136

McLaughlin, D. E., \& van der Marel, R. P. 2005, ApJS, 151, 304

Milone, A. P., Bedin, L. R., Piotto, G., et al. 2008, ApJ, 673, 241

Milone, A., Piotto, G., Bedin, L., et al. 2012, ApJ, 744, 58

Milone, A. P., Marino, A. F., Piotto, G., et al. 2013, ApJ, 767, 120

Momany, Y., Cassisi, S., Piotto, G., et al. 2003, A\&A, 407, 303

Nataf, D. M., Gould, A., Pinsonneault, M. H., \& Stetson, P. B. 2011, ApJ, 736, 94

Norris, J. 1981, ApJ, 248, 177

Paust, N. E. Q., Reid, I. N., Piotto, G., et al. 2010, AJ, 139, 476

Piotto, G., Milone, A. P., Anderson, J., et al. 2012, ApJ, 760, 39

Recio-Blanco, A., Aparicio, A., Piotto, G., De Angeli, F., \& Djorgovski, S. G. 2006, A\&A, 452, 875

Sbordone, L., Salaris, M., Weiss, A., \& Cassisi, S. 2011, A\&A, 534, A9

Shetrone, M. D., \& Keane, M. J. 2000, AJ, 119, 840

Skrutskie, M. F., Cutri, R. M., Stiening, R., et al. 2006, AJ, 131, 1163

Smith, G. H. 1983, AJ, 88, 410

Smith, G. H. 1984, AJ, 89, 1545

Smith, G. H., \& Langland-Shula, L. E. 2009, PASP, 121, 1054

Smith, V. V., Shetrone, M. D., \& Keane, M. J. 1999, ApJ, 516, L73

Székely, P., Kiss, L. L., Jackson, R., et al. 2007, A\&A, 463, 589

Valenti, J. A., Piskunov, N., \& Johns-Krull, C. M. 1998, ApJ, 498, 851

Vesperini, E., McMillan, S. L. W., D’Antona, F., \& D'Ercole, A. 2010, ApJ, 718, 112

Villanova, S., Geisler, D., \& Piotto, G. 2010, ApJ, 722, 118

Worley, C. C., \& Cottrell, P. L. 2010, MNRAS, 406, 2504

Yong, D., \& Grundahl, F. 2008, ApJ, 672, L29

Yong, D., Grundahl, F., Nissen, P. E., Jensen, H. R., \& Lambert, D. L. 2005, A\&A, 438, 875 


\section{Appendix A: Error estimates}

We refer the reader to the analogous Appendices in Carretta et al. (2009a,b) for a detailed discussion of the procedure adopted for error estimates. In the following, we only provide the main tables of sensitivities of abundance ratios to the adopted errors in the atmospheric parameters and $E W \mathrm{~s}$ and the final estimates of internal and systematic errors for all species analysed from UVES and GIRAFFE spectra of stars in NGC 362.

The sensitivities of derived abundances on the adopted atmospheric parameters were obtained by repeating our abundance analysis by changing only one atmospheric parameter each time for all stars in NGC 362 and by taking the average value of the slope change vs. abundance. This exercise was done separately for both UVES and GIRAFFE spectra.

We notice that the values usually adopted (determined from the scatter of abundances from individual lines) are overestimated when estimating the contribution to internal errors due to $E W \mathrm{~s}$ and $v_{\mathrm{t}}$, because regularities in the data are not considered.
These regularities are due to uncertainties in the $g f$-values, unrecognised blends with adjacent lines, inappropriate positioning of the continuum, etc. They show up in uniform deviations of individual lines from average abundances for each star. By averaging over all stars the residuals of abundances derived from individual lines with respect to the average value for each star, we estimated that some $36 \%$ of the total variance in the Fe abundances from individual lines is due to systematic offsets between different lines, which repeat from star-to-star. For about $30 \%$ of the line, these offsets have trends with temperature significant at about $2 \sigma$ level. However, we found that the additional fraction of variance that can be explained by these trends is very small, and we can neglect it. We conclude that the errors in $E W \mathrm{~s}$ and $v_{\mathrm{t}}$ should be multiplied by 0.8 when considering star-to-star variations (internal errors, according to our denomination).

The amount of the variations in the atmospheric parameters is shown in the first line of the headers in Tables A.1 and A.2, whereas the resulting response in abundance changes of all elements (the sensitivities) are shown in columns from 3 to 6 in these tables. 
E. Carretta et al.: Multiple stellar populations in NGC 362

Table A.1. Sensitivities of abundance ratios to variations in the atmospheric parameters and to errors in the equivalent widths, and errors in abundances for stars in NGC 362 observed with UVES.

\begin{tabular}{|c|c|c|c|c|c|c|c|c|}
\hline Element & $\begin{array}{c}\text { Average } \\
n . \text { lines }\end{array}$ & $\begin{array}{l}T_{\text {eff }} \\
(\mathrm{K})\end{array}$ & $\begin{array}{l}\log g \\
(\operatorname{dex})\end{array}$ & $\begin{array}{c}{[\mathrm{A} / \mathrm{H}]} \\
(\mathrm{dex})\end{array}$ & $\begin{array}{c}v_{\mathrm{t}} \\
\mathrm{km} \mathrm{s}^{-1}\end{array}$ & $\begin{array}{l}E W s \\
(\operatorname{dex})\end{array}$ & $\begin{array}{c}\text { Total } \\
\text { internal }\end{array}$ & $\begin{array}{c}\text { Total } \\
\text { systematic }\end{array}$ \\
\hline Variation & & 50 & 0.20 & 0.10 & 0.10 & & & \\
\hline Internal & & 5 & 0.04 & 0.05 & 0.04 & 0.087 & & \\
\hline Systematic & & 59 & 0.06 & 0.05 & 0.01 & & & \\
\hline$[\mathrm{Fe} / \mathrm{H}] \mathrm{I}$ & 81 & +0.041 & +0.016 & +0.003 & -0.033 & +0.010 & 0.017 & 0.051 \\
\hline$[\mathrm{Fe} / \mathrm{H}] \mathrm{II}$ & 9 & -0.051 & +0.104 & +0.034 & -0.012 & +0.029 & 0.041 & 0.072 \\
\hline$[\mathrm{O} / \mathrm{Fe}] \mathrm{I}$ & 2 & -0.029 & +0.067 & +0.032 & +0.030 & +0.062 & 0.066 & 0.068 \\
\hline$[\mathrm{Na} / \mathrm{Fe}] \mathrm{I}$ & 3 & +0.008 & -0.048 & -0.032 & +0.019 & +0.050 & 0.054 & 0.053 \\
\hline$[\mathrm{Mg} / \mathrm{Fe}] \mathrm{I}$ & 3 & -0.010 & -0.015 & -0.006 & +0.018 & +0.050 & 0.051 & 0.017 \\
\hline [Al/Fe]I & 2 & +0.003 & -0.024 & -0.010 & +0.028 & +0.062 & 0.063 & 0.052 \\
\hline$[\mathrm{Si} / \mathrm{Fe}] \mathrm{I}$ & 9 & -0.053 & +0.023 & +0.010 & +0.025 & +0.029 & 0.032 & 0.064 \\
\hline$[\mathrm{Ca} / \mathrm{Fe}] \mathrm{I}$ & 16 & +0.021 & -0.030 & -0.018 & -0.016 & +0.022 & 0.025 & 0.027 \\
\hline$[\mathrm{Sc} / \mathrm{Fe}] \mathrm{II}$ & 7 & +0.042 & -0.023 & -0.003 & -0.016 & +0.033 & 0.034 & 0.052 \\
\hline$[\mathrm{Ti} / \mathrm{Fe}] \mathrm{I}$ & 9 & +0.054 & -0.019 & -0.018 & -0.001 & +0.029 & 0.031 & 0.065 \\
\hline$[\mathrm{Ti} / \mathrm{Fe}] \mathrm{II}$ & 1 & +0.030 & -0.019 & -0.005 & +0.004 & +0.087 & 0.087 & 0.038 \\
\hline [V/Fe]I & 9 & +0.068 & -0.015 & -0.013 & +0.001 & +0.029 & 0.031 & 0.081 \\
\hline$[\mathrm{Cr} / \mathrm{Fe}] \mathrm{I}$ & 2 & +0.037 & -0.020 & -0.028 & -0.010 & +0.062 & 0.064 & 0.048 \\
\hline$[\mathrm{Cr} / \mathrm{Fe}] \mathrm{II}$ & 16 & +0.014 & -0.025 & -0.020 & +0.008 & +0.022 & 0.025 & 0.026 \\
\hline$[\mathrm{Mn} / \mathrm{Fe}] \mathrm{I}$ & 3 & +0.021 & -0.013 & -0.009 & -0.018 & +0.050 & 0.051 & 0.027 \\
\hline$[\mathrm{Co} / \mathrm{Fe}] \mathrm{I}$ & 5 & -0.008 & +0.002 & +0.003 & +0.024 & +0.039 & 0.040 & 0.019 \\
\hline$[\mathrm{Ni} / \mathrm{Fe}] \mathrm{I}$ & 29 & -0.014 & +0.016 & +0.008 & +0.014 & +0.016 & 0.018 & 0.019 \\
\hline$[\mathrm{Cu} / \mathrm{Fe}] \mathrm{I}$ & 1 & +0.010 & +0.019 & -0.003 & -0.017 & +0.100 & 0.103 & 0.091 \\
\hline$[\mathrm{Y} / \mathrm{Fe}] \mathrm{II}$ & 2 & +0.040 & -0.037 & -0.006 & +0.008 & +0.100 & 0.087 & 0.050 \\
\hline$[\mathrm{Zr} / \mathrm{Fe}] \mathrm{II}$ & 2 & +0.041 & -0.017 & -0.005 & +0.002 & +0.040 & 0.087 & 0.067 \\
\hline$[\mathrm{Ba} / \mathrm{Fe}] \mathrm{II}$ & 3 & +0.071 & -0.030 & -0.002 & -0.088 & +0.010 & 0.062 & 0.097 \\
\hline$[\mathrm{La} / \mathrm{Fe}] \mathrm{II}$ & 1 & +0.051 & -0.071 & +0.000 & -0.001 & +0.100 & 0.087 & 0.073 \\
\hline$[\mathrm{Ce} / \mathrm{Fe}] \mathrm{II}$ & 2 & +0.041 & -0.017 & -0.006 & +0.002 & +0.050 & 0.087 & 0.071 \\
\hline$[\mathrm{Nd} / \mathrm{Fe}] \mathrm{II}$ & 7 & +0.065 & -0.021 & -0.001 & +0.008 & +0.042 & 0.087 & 0.065 \\
\hline$[\mathrm{Eu} / \mathrm{Fe}] \mathrm{II}$ & 2 & +0.051 & -0.037 & -0.001 & +0.001 & +0.062 & 0.062 & 0.055 \\
\hline$[\mathrm{Dy} / \mathrm{Fe}] \mathrm{II}$ & 1 & +0.031 & -0.024 & -0.004 & +0.002 & +0.050 & 0.062 & 0.097 \\
\hline
\end{tabular}

Table A.2. Sensitivities of abundance ratios to variations in the atmospheric parameters and to errors in the equivalent widths, and errors in abundances for stars in NGC 362 observed with GIRAFFE.

\begin{tabular}{lcccccccc}
\hline \hline Element & $\begin{array}{c}\text { Average } \\
\text { n. lines }\end{array}$ & $\begin{array}{c}T_{\text {eff }} \\
(\mathrm{K})\end{array}$ & $\begin{array}{c}\log g \\
(\mathrm{dex})\end{array}$ & $\begin{array}{c}{[\mathrm{A} / \mathrm{H}]} \\
(\mathrm{dex})\end{array}$ & $\begin{array}{c}v_{\mathrm{t}} \\
\mathrm{km} \mathrm{s}^{-1}\end{array}$ & $\begin{array}{c}E W \mathrm{~s} \\
(\mathrm{dex})\end{array}$ & $\begin{array}{c}\text { Total } \\
\text { internal }\end{array}$ & $\begin{array}{c}\text { Total } \\
\text { systematic }\end{array}$ \\
\hline Variation & & 50 & 0.20 & 0.10 & 0.10 & & & \\
Internal & & 5 & 0.04 & 0.04 & 0.12 & 0.126 & & \\
Systematic & & 59 & 0.06 & 0.06 & 0.01 & & & \\
\hline$[\mathrm{Fe} / \mathrm{H}] \mathrm{I}$ & 29 & +0.052 & +0.000 & -0.005 & -0.028 & +0.023 & 0.041 & 0.062 \\
{$[\mathrm{Fe} / \mathrm{H}] \mathrm{II}$} & 2 & -0.033 & +0.091 & +0.023 & -0.011 & +0.089 & 0.093 & 0.048 \\
{$[\mathrm{O} / \mathrm{Fe}] \mathrm{I}$} & 1 & -0.041 & +0.081 & +0.034 & +0.031 & +0.126 & 0.133 & 0.059 \\
{$[\mathrm{Na} / \mathrm{Fe}] \mathrm{I}$} & 2 & -0.011 & -0.031 & -0.012 & +0.018 & +0.089 & 0.092 & 0.031 \\
{$[\mathrm{Mg} / \mathrm{Fe}] \mathrm{I}$} & 2 & -0.018 & -0.007 & -0.002 & +0.016 & +0.089 & 0.091 & 0.022 \\
{$[\mathrm{Si} / \mathrm{Fe}] \mathrm{I}$} & 6 & -0.046 & +0.025 & +0.010 & +0.027 & +0.051 & 0.061 & 0.055 \\
{$[\mathrm{Ca} / \mathrm{Fe}] \mathrm{I}$} & 5 & +0.007 & -0.024 & -0.006 & -0.010 & +0.056 & 0.058 & 0.011 \\
{$[\mathrm{Sc} / \mathrm{Fe}] \mathrm{II}$} & 4 & -0.053 & +0.080 & +0.030 & +0.011 & +0.063 & 0.068 & 0.067 \\
{$[\mathrm{Ti} / \mathrm{Fe}] \mathrm{I}$} & 3 & +0.023 & -0.008 & -0.008 & +0.009 & +0.073 & 0.074 & 0.027 \\
{$[\mathrm{~V} / \mathrm{Fe}] \mathrm{I}$} & 4 & +0.037 & -0.006 & -0.008 & +0.011 & +0.063 & 0.065 & 0.044 \\
{$[\mathrm{Cr} / \mathrm{Fe}] \mathrm{I}$} & 3 & -0.029 & -0.045 & -0.043 & -0.021 & +0.073 & 0.080 & 0.037 \\
{$[\mathrm{Co} / \mathrm{Fe}] \mathrm{I}$} & 1 & +0.005 & +0.011 & +0.007 & +0.022 & +0.126 & 0.129 & 0.018 \\
{$[\mathrm{Ni} / \mathrm{Fe}] \mathrm{I}$} & 6 & -0.010 & +0.015 & +0.006 & +0.020 & +0.051 & 0.057 & 0.014 \\
{$[\mathrm{Ba} / \mathrm{Fe}] \mathrm{II}$} & 1 & -0.038 & +0.057 & +0.037 & -0.057 & +0.126 & 0.145 & 0.055 \\
\hline
\end{tabular}

